\title{
COMPUTERIZED TOMOGRAPHY USING VIDEO RECORDED FLOUROSCOPIC IMAGES
}

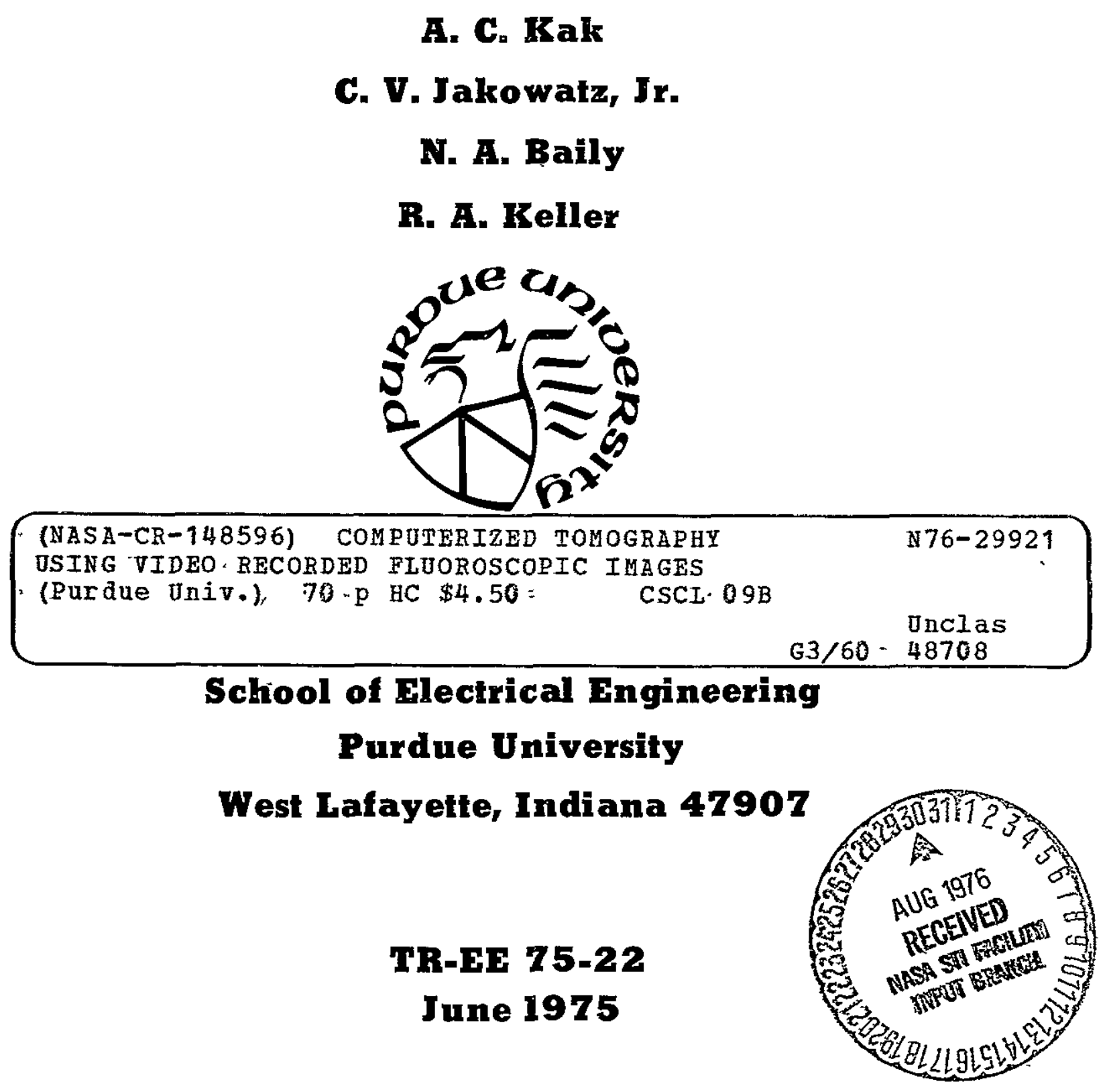

Work at Purdue University was supported by ICFAR, Regenstrief Institute for Health care and ARPA Grant F 30602-75-C-0150.

Work at University of Calıfornia, San Diego, was supported by NASA Grant NGR 05-009-257. 


\section{COMPUTERIZED TOMOGRAPHY USING VIDEO \\ RECORDED FLOUROSCOPIC IMAGES}

A. C. Kak

C. V. Jakowatz, Jr.

N. A. Baily

R. A. Keller

\section{TR-EE $\quad 75-22$ \\ June 1975}

\section{School of Electrical Engineering Purdue University \\ West Lafayette, Indiana 47907}

Work at Purdue University was supported by ICFAR, Regenstrief Institute for Health care and ARPA Grant F 30602-75-C-0150.

Work at University of California, San Diego, was supported by NASA

Grant NGR 05-009-257. 


\section{COMPUTERIZED TOMOGRAPHY USING VIDEO}

\section{RECORDED FLUOROSCOPIC IMAGES}

\section{A. C. Kak ${ }^{*}$, C. V. Jakowatz, Jr. ${ }^{*}$, N. A. Baily ${ }^{\dagger}$ and R. A. Keller ${ }^{\dagger}$}

In this paper we investigate the possibility of constructing computerized tomograms using data collected from a fluoroscopic system. It is shown that through proper handling of this data, useful images can be obtained. The system offers the advantages of eliminating the need for a highly stabilized, linear translating mechanism, and also of requiring relatively low patient dosage. In addition, the data gathering can be done in essentially real time.

Several significant problems arise when fluoroscopic data, gathered via an image intensifier/vidicon combination, is used for tomographic purposes. First, it turns out that it is difficult in such a system to accurately determine a value for the reference $x$-ray intensity. This is due to scatter and to the fact that optimal use of the quantizer range is desired. We have shown theoretcally. that the effects of such an error are to introduce a ring-like artifact into the reconstructed tomogram, and have shown how this problem may be eliminated. Second, a very important consideration with the fluoroscopic data is that no more than sixteen gray levels can be meaningfully recorded directly by the vidicon. This low signal-to-noise ratio would clearly not be sultable for

\footnotetext{
School of Electrical Engineering, Purdue Univ. W. Lafayette, IN 47907

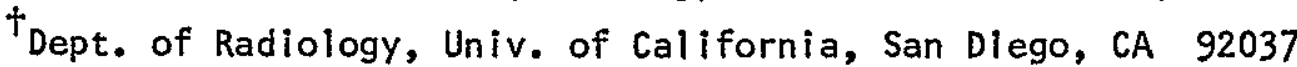
Work at Purdue University was supported by ICFAR, Regenstrief Institute for Health care and ARPA Grant F 30602-75-C-0150. Work at University of California, San Diego, was supported by NASA Grant NGR 05-009-257.
} 
reconstructions of tissue density differences on the order of one percent. However, since a large number of points, say 500, may be digitized from a given TV line, and since a frame may be recorded in only a few milliseconds time, spatial and temporal averaging may be performed to reduce considerably the noise variance, without suffering significant loss of spatial resolution. This allows accurate reconstructions to be done. Finally, the fact that a diverging radiation source is employed must be taken into account. We have demonstrated that one can avoid having to either rearrange data or develop efficient algorithms that directly handle a fan beam geometry by simply using a parallel ray convolution/back-projection algorithm, when the beam divergence is less than about $15^{\circ}$. Through computer simulations we have demonstrated these effects. In addition, we have reported results from our studies done on lucite phantoms and a freshly sacrificed rat. 
1. INTRODUCTION

The problem of digitally reconstructing the internal structures of an object from measurements of its two dimensional projections, resulting from transmission of radiation through the object, has been of interest in mathematics, radio-astronomy, and biological sciences for over 50 years. About four years ago this problem suddenly assumed major importance in medical engineering after Hounsfield and his associates [23] announced the development of a sophisticated computerized $x$-ray scanning system capable of reconstructing cross-sectional images (tomograms) of the human head with high tissue-density discrimination capability. This development was heralded as a major breakthrough in diagnostic radiology because it, for the first time, permitted noninvasive visualization of the ventricles of the brain, as well as a large class of brain tumors and injuries. Hounsfield's scanning system, also known as the EMI scanner, does have one disadvantage, however. Each projection required for constructing the tomogram is obtained by linearly moving a highly collimated $x$-ray beam across the body section (Fig. 1). This means that movements of $x \overline{-r a y}$ tube and the crystal detector have to be highly stabilized. This adds considerably to the cost of the system, which at present is beyond the reach of many medical institutions. This may also lead to poor quality tomograms if the object or parts of it are in excessive motion during each linear scan, such as may be the case in attempting to image the heart.

In this paper we have examined a computerized tomographic imaging system which employs video-recorded fluoroscopic images as input data. By hooking the video recorder to a digital computer through a suitable interface, such a system permits very rapid construction of tomograms. In fact, the system can be made in essence "real time". With the use of fluoroscopic images, the tomographic imaging system is essentially equivalent to the one 
shown in Fig. 2. Note that an entire projection is now recorded in a single instant of time, reducing the total time required for all of the data collection. Also, if the object now changes in time and if these changes are periodic, different projections can be taken at time instances synchronized with periodic variations, so that the reconstructed tomogram will represent the cross-sectional slice of the object at one instant of time.

While computerized tomography using fluoroscopic data does have the advantages of requiring lesser data collection time and being immune to periodic variations in the object structure than the more familiar system requiring the linear motion, it does suffer from the disadvantage of the inherently low signal to noise ratio present in fluoroscopic images. As was shown earlier [3] the signal to noise ratio problem can be alleviated by suitable spatial and temporal averaging of the data.

It is clear that both techniques for data collection, the linear motion of the source-detector in the EMI machine and the video recording of fluoroscopic images, are equally important for computerized tomography. While the superior signal-to-noise ratio in the former are ideal for imaging the human head where good tissue discrimination capability is required, the latter technique is superior for imaging parts like the thorax where periodic variations in the internal structure occur.

In addition to the low signal to noise ratio, the other major distortions present in the fluoroscopic data arise from: the approximately logarithmic non-linearity introduced by the fluoroscopic screen; errors in recording the air-transmission values, which result in the presence of ring-like artifacts when convolution algorithms are used for reconstruction; and the fact that projections are taken by non-parallel radiation. A recent paper [34] dealt with the problem of non-parallel radiation by using an algebraic reconstruction 
algorithm in which simultaneous equations were formed to explicitly take into account the non-parallel nature of rays. However, the algebraic techniques in general are known to be less accurate than the convolution techniques." Although the development of convolutional techniques for projections taken with radially diverging radiation has recently been reported, such algorithms seem to require large computer time. In this paper we have shown that for beams with divergence up to approximately 15 degrees one can use the convolution algorithm designed for the parallel radiation case with negligible degradation both quantitatively and from the point of view of visual quality. In fact, it may be shown that for very small angles of beam divergence a convolutional algorithm designed for the parallel beam case gives superior results and is computationally more efficient than either the algebraic techniques or the convolutional algorithms for radially diverging data.

In the next section we will first very briefly review the i iterature dealing with digital reconstruction of objects from their projections and then discuss the digital implementation of the convolution algorithm.

\footnotetext{
*This statement must be taken with some caution. The speed and the accuracy. of an algorithm is dependent upon the pattern that needs to be reconstructed. Our conclusions are based on the comparisons made by many researchers on phantoms relevant to x-ray computerized tomography in medicine. For example, for the phantoms of the human head section, Shepp and Logan [33] compared the parallel ray convolution algorithm with the algebraic technique and concluded that the algebraic algorithm required considerably more computing time to obtain a reconstruction of comparable accuracy.
} 
2. RECONSTRUCTION OF OBJECTS FROM THEIR PROJECTIONS

Let $f(x, y)$ be a two dimensional function shown in Fig. 3 . Let the integration of the function $f(x, y)$ along lines subtending angles of $\theta+90^{\circ}$ with the x-axis be denoted by $P_{\theta}(t)$ where $t$ is a distance parameter as shown in the figure. The $P_{\theta}(t)$ is called the projection of the function at an angle $\theta$. The problem of reconstructing an object from its projections is then equivalent to reconstructing $f(x, y)$ from a knowledge of $P_{\theta}(t)$ for various $\theta$.

Many investigators $[4-9,11-22,24-30,33,35]$ have addressed themselves to the above problem. A number of different approaches have been developed, the more important falling in three categories: the algebraic methods [16-18,20-22], the fourier methods, $[1]-15$, $26,28,35]$ and the convolution or the filtered back-projection methods $[4,5,7-9,24,29,33]$. Of these techniques, the convolution or the filtered back-projection methods are known to be computationalily most efficient and accurate. We will now discuss the digital implementation of the techniques that fall in this category.

Let, $S_{\theta}(u)$ be the Fourier transform of the projection $P_{\theta}(t)$, that is

$$
S_{\theta}(u)=\int_{-\infty}^{\infty} p_{\theta}(t) e^{-j 2 \pi u t} d t
$$

Given $S_{\theta}(u)$ for all $\theta$, it is known that the two dimensional

function ' $f(x, y)$ (Fig. 3) can be reconstructed by using the following relationship

$$
f(x, y)=\int_{0}^{\pi} \Omega_{\theta}(x \cos \theta+y \sin \theta) d \theta
$$


where

$$
Q_{\theta}(t)=\int_{-\infty}^{\infty} S_{\theta}(u)|u| e^{j 2 \pi u t} d u
$$

The above formulas for reconstruction say that from each projection $P_{\theta}(t)$ we calculate a "filtered projection" $Q_{\theta}(t)$ by using (1) and (3), and use (2) to reconstruct the function $f(x, y)$.

The parameter $u$ has the dimension of spatial frequency. The integration in (3) must, in principle, be carried out over all the spatial frequencies. In practice the energy contained in the Fourier transform components above a certain frequency would be negligible. So for all practical purposes the projections may be considered to be bandlimited, an argument used by Mersereau and Oppenheim [27] to arrive at some interesting theoretical results. Next, let $W$ be the smallest frequency beyond which the spectral energy in all the projections may be ignored. Then by using the sampling theorem [32], the projection can be represented by

$$
P_{\theta}(t)=\sum_{k=-\infty}^{\infty} P_{\theta}\left(\frac{k}{2 W}\right) \frac{\sin 2 \pi W\left(t-\frac{k}{2 W}\right)}{2 \pi W\left(t-\frac{k}{2 W}\right)}
$$

Substituting (4) in (1), we get

$$
S_{\theta}(u)=\frac{1}{2 W} \sum_{k=-\infty}^{\infty} P_{\theta}\left(\frac{k}{2 W}\right) e^{-j 2 \pi u \frac{k}{2 W} b_{W}(u)}
$$

where

$$
\begin{aligned}
b_{W}(u) & =1 & & |u| \leq w \\
& =0 & & \text { otherwise. }
\end{aligned}
$$


Now if the projection functions are of finite order [27], which means they can be represented by $N+1$ samples for some value of $N$, (5) reduces to

$$
S_{\theta}(u)=\frac{1}{2 W} \sum_{k=-N / 2}^{N / 2} P_{\theta}\left(\frac{\dot{k}}{2 W}\right) e^{-j 2 \pi u \frac{k}{2 W} b_{W}(u)}
$$

We will arbitrarily assume $N$ to be an even number. Now the functions $S_{\theta}(u)$ are zero outside the interval $(-W, W)$ of the spatial frequency axis. Suppose that in this interval we desire to know each. $S_{\theta}(u)$ at a set of equispaced points given by

$$
u=m \frac{2 W}{N} \text { for } m=-\frac{N}{2}, \ldots, 0, \ldots, \frac{N}{2} \text {. }
$$

Substituting these in (6), we get

$$
\begin{aligned}
S_{\theta}\left(m \frac{2 W}{N}\right)=\frac{1}{2 W} \sum_{k=-N / 2}^{N / 2} P_{\theta}\left(\frac{k}{2 W}\right) e^{-j 2 \pi \frac{m k}{N}} \\
\quad m=-\frac{N}{2}, \ldots, 0, \ldots, \frac{N}{2}
\end{aligned}
$$

The above equation is the familiar discrete Fourier transform (DFT) relationship and, therefore, can be rapidly evaluated by the fast Fourier transform (FFT) algorithms.

Given the samples of a projection, the equation (7) gives the samples of $i$ ts Fourier transform. The next step is to evaluate the "modified projection" $Q_{\theta}(t)$ digitally. Since the Fourier transforms $S_{\theta}(u)$ are bandlimited, (3) can be approximated by

$$
Q_{\theta}(t)=\int_{-W}^{W} s_{\theta}(u)|u| e^{j 2 \pi u t} d u
$$




$$
\simeq \frac{2 W}{N} \sum_{m=-N / 2}^{N / 2} s_{\theta}\left(m \frac{2 W}{N}\right)\left|m \frac{2 W}{N}\right| e^{j 2 \pi m \frac{2 W}{N} t}
$$

provided $N$ is large enough. Again, if we say that we would like to know the modified projections $Q_{\theta}(t)$ for only those $t$ at which the projections $P_{\theta}(t)$ are sampled, we get

$$
\begin{aligned}
Q_{\theta}\left(\frac{k}{2 W}\right) \simeq\left(\frac{2 W}{N}\right) \sum_{m=-N / 2}^{N / 2} s_{\theta}\left(m \frac{2 W}{N}\right)\left|m \frac{2 W}{N}\right| e^{j 2 \pi m \frac{m k}{N}} \\
k=-N / 2, \ldots,-1,0,1, \ldots, N / 2
\end{aligned}
$$

By the above equation the function $Q_{\theta}(t)$ at the sampling points of the projection function is given by the inverse DFT of the product of $S_{\theta}\left(m \frac{2 W}{N}\right)$ and $\left|m \frac{2 W}{N}\right|$. By the familiar convolution theorem for the case of discrete transforms, (10) can be written as

$$
Q_{\theta}\left(\frac{k}{2 W}\right) \simeq \frac{2 W}{N} P_{\theta}\left(\frac{k}{2 W}\right) * \phi\left(\frac{k}{2 W}\right)
$$

where * denotes the operation of circular convolution and where $\phi\left(\frac{k}{2 W}\right)$ is the inverse DFT of the discrete function $\left|\frac{2 W}{N}\right|, m=-\frac{N}{2}$, $\ldots,-1,0,1, \ldots, \frac{\mathrm{N}}{2}^{\dagger}$

Clearly at the sampling points of the projections, the functions $Q_{\theta}(t)$ may be obtained either in the Fourier domain by using $(10)$, or in the signal domain by using (11). The reconstructed picture $f(x, y)$ may then be obtained by the discrete approximation to the integral in (2), i.e.,

\footnotetext{
In practice superior results are usually obtained by padding the projection data with a sufficient number of zeroes so that for $k=-N / 2$, $\ldots \ldots, N / 2$ the modified projection is equal to the aperiodic convolution of the projection data and filter impulse response. This is because in practice the assumptions of finite bandwidth and finite order are not strictly satisfied.
} 


$$
\hat{f}(x, y)=\frac{\pi}{K} \sum_{i=1}^{K} Q_{\theta_{i}}\left(x \cos \theta_{i}+y \sin \theta_{i}\right)
$$

where the $K$ angles $\theta_{i}$ are those for which the projections $P_{\theta}(t)$ are known。

Equation (12) calls for each filtered projection $Q_{\theta_{i}}$ (t) to be "back-projected". This can be explained as follows. To every point $(x, y)$ in the image plane there corresponds a value of $t \Leftrightarrow x \cos \theta$ $+y \sin ^{2} \theta$ ). for a'given value of $\theta_{0}$. The contribution that $Q_{\theta_{i}}$ makes to the reconstruction at $(x, y)$ is the value of $Q_{\theta_{i}}$ for the corresponding value of $t$. This is further illustrated in Fig. 4. It is easily shown that for the indicated angle $\theta_{i}$, the value of $t=\left(x \cos \theta_{i}+y \sin \theta_{i}\right)$ is same for all $(x, y)$ on the line LM. Therefore, the filtered projection $Q_{\theta_{i}}$ will make the same contribution to the reconstruction at all these points. From this follows that in reconstruction each function $Q_{\theta_{i}}(t)$ is smeared back over the image plane. The sum (multiplied by $\pi / K)$ of all such smearings results in the reconstructed image. Note that the value of $x \cos \theta_{i}+y^{\prime} \sin \theta_{i}$ in (12) may not correspond to one of the values of $t$ for which $Q_{\theta_{i}}$ is determined in (10) or in (11). However, $Q_{\theta_{i}}$ for such $t$ may be approximated by interpolation. We have used 1 inear interpolation, which naturally introduces some errors since $Q_{\theta_{i}}(t)$, in general, is not linearly dependent upon $t$. The reconstruction technque discussed here was tested by computer simulation on a number of test patterns. One such pattern is shown in Fig. 5a which is obtained by superimposing ellipses. The advantage of using ellipses for computer simulation was first pointed out by Shepp and Logan [33]. This relies on the fact that one can write down simple analytic expressions for the projections of such patterns. 
Note that projections obtained in this way are the "real-projections" as opposed to "pseudo-projections" [21]. In Fig。5a there are fine gray level differences between various regions. However, these cannot be seen in the photograph. Fig. $5 c$ illustrates some of these differences. The heavy dotted line in Fig. $5 c$ shows gray levels across the middle horizontal section in Fig。 5 a.

Sixty projections of the patterns in Fig. 5 a were calculated by the method in Appendix A。 Each projection was sampled at 101 points. The image reconstructed from this data by using equations (11) and (12) is shown in Fig. 5b. The light solid line in Fig. 5c shows the gray levels across the middle horizontal section in the reconstruction in Fig.5b。 


\section{A SYSTEM FOR COMPUTERIZED TOMOGRAPHY USING FLOUROSCOPIC DATA}

Figure 6 shows a block schematic of the system used for the collection of the projection data. The phantom was mounted on a turntable. For each position of the turntable two frames of the projected image of the phantom were recorded on the video disc by the image-intensifier vidicon combination. Because of the high rate at which the data is produced by the vidicon, it cannot be directly digitized. The use of the video disc and the scan converter is to slow down the data rate before its digitization by the $A / D$ converter.

Only a small area of each TV frame (Fig. 7) recorded on the video disc is actually digitized. The vertical width of this digitized area is approximately equal to the thickness of the crosssectional slice of the phantom for which the tomogram is constructed. As shown in Fig. 7, a strip of aluminum is attached to the face of the flouroscopic screen of the image-intensifier so that a part of the digitized data for each projection is a measure of the x-ray transmission through aluminum. These values are used for scan-to-scan normalization of the data against variations in the $x$-ray generation. Note that the aluminum strip is outside the image of the phantom on the image intensifier. Aluminum is used to prevent the saturation of the flourescent screen by $x$-rays that travel directly from the $x$-ray source to the screen.

The part of each TV frame that is digitized, as shown in Fig. 7 , is sampled on a $240 \times 8$ grid of points, 240 horizontally and 8 vertically. After digitization, spatial averaging is performed over blocks of $3 \times 8$ points ( 3 horizontally and 8 vertically), so that 
each $240 \times 8$ record reduces to a string of 80 data values. The regional averaging performed reduces such noise as that generated by quantum mottle, the image amplifier - TV chain, recording, playback and digitization. Temporal averaging further reduces the effects of nearly all types of noise. Temporal averaging was employed to a limited extent by recording two frames for each projection, and then averaging the results.

Each of the 80 data elements for each projection was represented by a 7 bit word. In other words, the quantization of the spatially and temporally averaged data was done to 128 levels. It should be noted that such a fine quantization has no meaning without the spatial and temporal averaging employed. That is because even under the best of laboratory conditions the flouroscopic data as directly recorded by the vidicon do not have more than fourteen gray levels $[1,2,10]$, implying a high statistical variance of the individual data points. As we have indicated later, the averaging employed reduces the noise variance of the data by a factor approximately equal to 48 .

By rotating the platform in Fig. 6 one can take projections of the test object at the desired number of angles, usually between 60 and 180. Of course, in a clinical version of the system, one would rotate the $x$-ray source and the detector combination around the patient, as opposed to rotating the object we have done. The two types of motions for generating projections are equivalent, however. In the next few sections we will by theory and/or computer simulation analyze the effects of some of the problems associated with flouroscopic data in the context of computerized tomography. 
4. LOGRITHMIC NON-LINEARITIES IN DATA AND THE EFFECTS OF ERRORS IN MEASUREMENT OF AIR-TRANSMISSTION VALUES

Consider a parallel beam of x-rays shown in Fig. $8 a$. Let $N_{0}$ be the intensity of the beam before transmission through the object. Evidently, $\mathrm{N}_{0}$ is proportional to the number of photons per unit area per unit time. Let us now consider the propagation of $x$-radiation along a particular ray labeled CD in Fig. 8a: In the absence of any absorption the intensity at $D$ is also equal to $\mathrm{N}_{0}$. However, in the presence of absorption by an object in the path of the beam, and under the assumption that the $x$-rays are monochromatic, the intensity at $D$ is given by the Lambert-Beer relationship

$$
N=N_{0} e^{-f_{C}^{D} \alpha(s) d s}
$$

where $\alpha(s)$ is the composite attenuation coefficient of the object along the path CD.

In a fluoroscopic imaging system the beam of $x$-radiation is fan shaped rather than parallel. Therefore, the $x$-ray intensity decreases not only as a result of absorption in-the material, but also due to beam divergence. The x-ray beam for our system can be approximated by that shown in Fig. 8b. Let the total number of photons emitted by the source 0 per unit time into the

\footnotetext{
*In practice the x-ray beams are polychromatic. The spectrum of a polychromatic beam changes continually as it propagates through an inhomogeneous medium. An interesting discussion on the difficulty that this causes in interpreting the numbers in a computerized tomogram can be found in [36]. Under the condition that the detector integrates all the transmitted photons, it has not yet been possible to incorporate the interaction of a polychromatic beam with an inhomogeneous medium in the theory of reconstruction of objects from their projections. it is for this reason various researchers in the theory of reconstruction have continued to make the assumption of monochromaticity. Note that in discussing their results for a phantom consisting of a number of $2.5 \mathrm{~cm}$ rods of commerical grade materials in a fixed-length water bath and $120 \mathrm{KeV}$ operation, the authors in [36] point out that the numbers produced by the EMI scanner give very closely the attenuation coefficients calculated for monochromatic x-rays of energy $73 \mathrm{KeV}$. The fact that the assumption of monochromaticity here does not give meaningless results is clear from the corroboration of the equation (31) by the ring like artifact in the tomogram derived from the experimental data in Fig. 17b.
} 
cone OPQRS be $N_{0}$. Let $N_{r}$ be the x-ray intensity at a distance $r$ in terms of the number of photons per unit area per unit time. clearly, under the assumption that $x$-ray emission within the cone has no angular dependence, the following result follows from the conservation of the total number of photons:

$$
r^{2} \Phi \in N_{r}=N_{0}
$$

Equivālently,

$$
N_{r}=\frac{N_{0}}{r^{2} \Phi \theta}
$$

Note that under the idealization that the radiation source can be considered to be a point source, ${ }_{r}$ should approach infinity as $r$ lends to zero.

Equation (14) gives the attenuation due to beam divergence of $x$-ray in tensity along a ray like OM in Fig。 $8 \mathrm{~b}$. If now an absorbing material is placed in the path of the ray $O M$ as in Fig. $8 \mathrm{c}$ the intensity at the point $M$ will be given by

$$
N_{r}=\frac{N_{0}}{r^{2} \Phi \Theta} e^{-\int_{0}^{M} \alpha(s) d s}
$$

where again $\alpha(s)$ is the attenuation coefficient of the material along the ray OM. For practical purposes it is more convenient to express the intensity at point $M$ in terms of the intensity at the point $A$ in Fig. $8 c$ for a ray like $0 A$ which does not pass through the object. Intensity values at points like $A$ in Fig. $8 c$ are called air-transmission intensities. Let $N_{A}$ be the air-transmission intensity. By $(14), N_{0}=N_{A} r^{2} \Phi$. Substituting this in (15), we get for the intensity at $M$

$$
N_{r}=N_{A} e^{-\int_{0}^{M} \alpha(s) d s}
$$


The composite attenuation coefficient $\alpha$ is in general a function of position in the absorbing material, that is, a function of the coordinates $x, y$, and $z$ shown in Fig. 8c. If the angle $\theta$ is small enough, the dependence on $z$ can be ignored and the attenuation can be considered to be essentially a two dimensional function $\alpha(x, y)$. Expressing this dependence explicitly, (16) can be written as

$$
N_{r}=N_{A} e^{-\int_{\text {ray }} O M} \alpha(x, y) d s
$$

Since in the system described in Section 3, the measurements in the strip PQRS in Fig. 8c are integrated along the shorter direction, we have essentially the two dimensional system shown in Fig. 8d, where we have ignored the curvature of the strip PQRS. It is clear from the above discussion that the measurement $I(t)$ at, say $M^{-}$, which is a distance $t$ from the indicated reference point $0^{\circ}$, is given by

$$
I(t)=I_{A^{-e}}-\int \alpha(t)=d s
$$

where $I_{A^{-}}$is the air-transmission measurement at a point like $A^{-}$and where $L(t)$ is the ray path from the source 0 to $M^{\circ}$ 。

The above relationship is valid only in the absence of any non-linearities introduced by the detection mechanism. However, it has been experimentally observed that the recorded measurements are approximately a logrithmic function of the $x$-ray intensity impinging on the image intensifier tube (see Fig. 9).* Therefore, over a large dynamic range what is actually measured is a quantity proportional to $\ln I(t)$. We get from (18)

$$
\ln I(t)=-f_{L(t)} \alpha(x, y) d s+\ln I_{A^{\prime}}
$$

\footnotetext{
*Further discussion on this can be found in "Response of Image Intensifier Fluoroscopic - TV System," by Baily and Keller to appear in INVESTIGATIVE RADIOLOGY.
} 
From Fig. $8 d$ it is clear that $\int_{L(t)} \alpha(x, y) d s$ is the projection of the function $\alpha(x, y)$ by a diverging beam on the line $P^{\prime} \cdot Q^{\prime}$. Evidently, projections at different angles may be taken by rotating the sourcedetector arrangement around the object. In the preceding section we used $P_{\theta}(t)$ to denote the projection at angle $\theta_{0}$ From (19), we get

$$
P_{\theta}(t)=-\ln I(t)+\ln I_{A^{\prime}}
$$

where the data $\ln 1(t)$, of course, depends upon the angle $\theta$ at which the projection is taken.

It is clear from $(20)$ that if $P_{\theta}(t)$ is to be known correctly, the air-transmission values $\ell_{n} I_{A}$ - must be known. Experience has shown that in practice $\ln A_{A}$, if taken simply from a ray like $O A^{\prime}$ in Fig. $8 \mathrm{c}$, is in general not known sufficiently accurately, the errors being large enough to cause the presence of structured artifacts in the tomograms. The errors in the measurement of $\ln \mathrm{I}_{A}$ - may be caused by one or both of the following factors.

(i) Limitations of the quantizer in the digitization process [32]: The output of the scan converter (see Section 3) consists of samples that may take any value in a continuous range. The quantizer divides the continuous range into a fixed number of intervals and all the samples with values in an interval are assigned a fixed value at the output of the quantizer (Fig。 10). Evidently, the operation of the quantizer requires that the lower and the upper limits, $I_{\min }$ and $I_{\max }$, respectively, of the continuous range be specified. To minimize the quantization error for a given number of output levels, $I_{\max }$ must be close to the largest value and $I_{\min }$ close to the smallest value of the intensities that result after transmission through the object. If $I_{\max }$ is 
chosen close to the air-transmission values, all the useful information (i.e., rays that do go through the object) would get crowded toward the bottom end of the quantization scale, leading to large quantization distortion. This implies that the airtransmission values may in practice be much larger than $I_{\max }{ }^{*}$ Since for any value larger than ' max' the output of the quantizer is $q_{N}(F i g .10)$, there may be greater errors in recording airtransmission values than other data.

ii) Scattering: The interation between photons and matter results in the removal of the photons from the original beam either by scattering or by absorption. The attenuation of an $x$-ray beam caused by both these phenomena is represented by the coefficient $\alpha$ in equations (13) through (19). Some. scattered photons reach the flourescent screen of the image intensifier and cause the degradation of the signal to noise ratio there. Now if to reduce the noise variance, the air-transmission values are calculated by averaging the data for rays that do not pass through the object (after the field inhomogeneities have been subtracted out), it is clear that due to the non-negative nature of the contribution by scattering, this average will be greater than that in the absence of scattering.

We will now show theoretically that the errors in the airtransmission data ( $\ln \mathrm{I}_{A^{*}}$ in (20)) will lead to the presence of ring like structures in the reconstructed tomograms.

The equation (3) for filtered projections, $Q_{\theta}(t)$, can be written as 


$$
Q_{\theta}(t)=\frac{1}{2 \pi} \quad \int_{-\infty}^{\infty} j 2 \pi S_{\theta}(u)[-j \operatorname{sgn}(u)] e^{j 2 \pi u t} d u
$$

where the function $\operatorname{sgn}(u)$ denotes

$$
\begin{aligned}
\operatorname{sgn}(u) & =1 \text { for } u \geq 0 \\
& =-1 \text { for } u<0
\end{aligned}
$$

By using the standard convolution theorem (21) can be written as

$$
Q_{\theta}(t)=\left\{1 . F . T \text {. of } j 2 \pi u S_{\theta}(u)\right\} *\left\{1 . F . T \text { of } \frac{-j}{2 \pi} \operatorname{sgn}(u)\right\}
$$

where the symbol $*$ denotes convolution, and the abbreviation I.F.T. stands for inverse Fourier transform. The I.F.T. of $j 2 \pi u S_{\theta}(u)$ is $\frac{\partial}{\partial t} p_{\theta}(t)$ while the I,F.T. of $\frac{-j}{2 \pi} \operatorname{sgn}(u)$ is $\frac{1}{t}$. Therefore, (23) can be written as

$$
Q_{\theta}(t)=\frac{\partial P_{\theta}(t)}{\partial t} * \frac{1}{2 \pi^{2} t}
$$

Now, the second term on the R.H.S. in (20) is not a function of $t$. Also, note the air-transmission value, once it is determined, is same for all the projections. (The data is corrected for scanto-scan variations.) Therefore, errors in $\ln I_{A}$ - cause a d.c. shift in all of the projections. Let this d.c. shift be denoted by $d_{e}$. If $P_{\theta}(t)$ denotes the correct projection and $P_{\theta}{ }_{\theta}(t)$ the recorded projection in the presence of errors $\ln ^{~} \mathrm{~A}_{\mathrm{A}^{-}}$, it is clear

$$
P_{\theta}^{\prime}(t)=P_{\theta}(t)+d_{e}
$$

Since the reconstruction algorithm discussed in Section 2 is 1 inear, the tomogram made from projections $\mathrm{P}_{\theta}^{-}(t)$ obtained from the recorded data will be the sum of tomograms from the $P_{\theta}(t)$ and whatever results when each projection is taken to be a constant equal to $d_{e}$. 
Therefore, the distortion in the reconstructed tomogram is another tomographic image caused by $d_{e}$ in (25). If e $(x, y)$ denotes the distortions in the tomogram, clearly from (2) and (24)

$$
e(x, y)=\int_{0}^{\pi} Q_{\theta}^{\prime}(x \cos \theta+y \sin \theta) d \theta
$$

where

$$
Q_{\theta}^{\prime}(t)=\frac{\partial}{\partial t}\left(d_{e}\right) * \frac{1}{2 \pi^{2} t}
$$

For the purpose of illustration let us assume that the object is bounded by a circle of unit radius and that the data for all the projections is recorded over a length of 2 (i.e., for $t$ between -1 and +1$)$. The d.c. shift in each projection is then a rectangular pulse as shown in Fig.lla. The derivative of this function is shown in Fig. Ilb and can be expressed as

$$
\frac{\partial}{\partial t} d_{e}=d_{e}[\delta(t+1)-\delta(t-1)]
$$

Substituting this in-(27), we get

$$
\begin{aligned}
Q_{\theta}^{-}(t) & =d_{e}\left[\frac{1}{t+1}-\frac{1}{t-1}\right] \\
& =\frac{d e}{\pi^{2}\left(1-t^{2}\right)}
\end{aligned}
$$

Substituting (29) in (26), we get for the distortion $e(x, y)$ in the tomogram

$$
e(x, y)=\frac{1}{2 \pi^{2}} \int_{0}^{\pi} \frac{2 d}{1-(x \cos \theta+y \sin \theta)^{2}} d \theta
$$

The integration in (30) can be simplified by noting that the distortion caused by the error in the air-transmission value should 
be a circularly symmetric function. That is, if $r$ is the radial distance from the center of the picture, $e(x, y)$ should be function of the form $E(r)$ where $r=\sqrt{x^{2}+y^{2}}$. This function $E(r)$ can be obtained by putting $x=y$ in (30) and writing the L.H.S. as $E(r)$. We get:

$$
\begin{aligned}
E(r) & =\frac{1}{2 \pi^{2}} \int_{0}^{\pi} \frac{2 d e}{1-x^{2}(\cos \theta+\sin \theta)^{2}} d \theta \\
& =\frac{d e}{\pi} \frac{1}{\sqrt{1-r^{2}}}
\end{aligned}
$$

The above result shows that the error in the air-transmission value should result in the appearance of circularly symmetric artifact in the tomogram, the pattern being such that its intensity increases sharply near the outer edges of the tomogram. Even though in theory these ring like artifacts take infinitive values at the outer boundary of the picture, in actual digital implementation these will be finite, their magnitude depending upon the spatial sampling density in the picture plane.

In order to simulate the effects of errors in the air-transmission values, a constant value was added to all the projections of the pattern in Fig. 5a. Fig. 12 shows the reconstructed image when the value of this constant is approximately 30 percent of the maximum gray level difference in Fig. 5a. Note the circular artifact outside the main pattern in Fig. 12. This is in keeping with the theoretical conclusions just arrived at. Also note that even though from a qualitative (visual) standpoint the artifacts caused by air-transmission errors lie primarily outside the main pattern in Fig. 12, from the quantitative standpoint the distortion exists over the entire image, its form given by eq. (31). 
In section 7 artifacts of the type discussed here will be shown to occur in the tomograms of a phantom and of a thoracic section of a rat. Based on the discussion presented here, we will remove these artifacts by introducing a correction in the air-transmission value. 


\section{QUANTIZATION EFFECTS}

In general, the noise level present in the TV and video disc recorder, even under the best of laboratory conditions, allows a maximum of fourteen gray levels $[1-3,10]$. This represents a high noise content. If an attempt is made to achieve reconstructions from this data without any preprocessing, the resulting pictures would generally be of low quality.

To get around this problem of low signal to noise ratio, the spatial and temporal averaging described in Section 3 was employed. Each data element in the projections is obtained by spatially averaging 24 samples of the recorded images and temporally averaging over two recordings. With this averaging, the noise variance of the projection data should be approximately $\frac{1}{48}$ of the original noise variance, the result being strictly correct only if we can assume that the noise in each image sample is independent and identically distributed.

Because of the considerable reduction in the noise variance, the data obtained after spatial and temporal averaging should contain many more useful gray levels than the dozen or so in the fluoroscopic image. It was because of this reason that a 7-bit-quantizer was used, yielding 128 gray levels for the projection data.

The discussion here does bring to light the fact that if fluoroscopic data is used for reconstruction, the degree of quantization is dependent upon the extent of the spatial and temporal averaging employed.

It is clear that the degree of quantization has to be matched to the signal-to-noise ratio of the data. While overmuantization, in general, results in increased demands on the core and other peripheral memory requirements and, sometimes, reduced speeds in $A / D$ conversion, under-quantization always leads to deterioration of the reconstructed Image. To illustrate this effect by computer simulation, the samples of the analytic functions for the 
projections of the picture in Fig. 5a were quantized to 8 levels. The reconstructed tomogram with this quantization is shown in Fig. 13a. When the data is quantized to 16 levels, the reconstruction is shown in Fig. 13b. Note that this is approximately ${ }^{*}$ the picture that would result if in a fluoroscopic system no spatial or temporal averaging was employed because the number of useful levels in the data would only be approximately 16 . When the data is quantized to 32 and 128 levels, respectively, the reconstructed images are shown in Figs. $13 \mathrm{c}$ and $13 \mathrm{~d}$.

* We have said approximately because while the real fluoroscopic data has random noise, the quantization noise in the simulated data is more structured. The structured noise may generate artifacts by interference. A more realistic model would be to add random noise to the simulated data before or after quantization. 
6. EFFECTS OF DIVERGING RADIATION

In the system discussed in Section 3 the projections are taken with diverging radiation. One can take two approaches in constructing tomograms from such projections: (i) Rearrange the projection data in such a way that the new data is approximately what would be generated by a parallel beam of radiation, and then use an algorithm like that in Section 2 to make the tomogram; or (ii) Develop an algorithm that directly yields a tomogram from the projection data taken with diverging radiation.

In Fig. 14 we have shown how one may rearrange the projection data obtained with diverging radiation to generate approximately the projections that would result if a parallel beam source was used. For the position 0 , of the source, the projection data is recorded on the line $D_{1} D_{2}$. A different projection is obtained when the source is moved to $O^{\prime}$ and the detection line to $D_{1}{ }^{\prime} D_{2}$ '. The ray $O A$ in the first projection is parallel to the $0^{\circ} \mathrm{A}^{\circ}$ in the second projection. By grouping from all the recorded projections the rays that are parallel to, say, OA in Fig. 14 one can approximately obtain projection data that would be generated by a parallel beam of $x$-rays, parallel to the $O A$ line. One can then use the algorithm in Section 2 to construct the tomogram. The disadvantage of this technique is the additional computer time required for rearranging the data.

Clearly, it is desirable to have an algorithm that would directly convert the projections obtained with diverging radiation into tomograms. It is relatively easy to devise algebraic types of 
algorithms for this purpose [31]. However, algebraic reconstructions are inherently less accurate and computationally less efficient than the convolution type algorithms such as that discussed in Section 2. Convolution type algorithms for data obtained with diverging radiation are still under development at Purdue University and elsewhere and are not yet available for experimental work.

We will now show by computer simulation that for upto $15^{\circ}$ in beam divergence, if the convolution algorithm in Section 2 is used for constructing tomograms from projection data obtained with diverging radiation, the distortion introduced is negligible. For the pattern shown in $\mathrm{Fig}$. 5 a the computer was programmed to generate projection data using diverging rays by the method discussed in Appendix A. The projection data was generated for the following cases of beam divergence: $5^{\circ}, 10^{\circ}, 15^{\circ}, 20^{\circ}$, and $60^{\circ}$. In each case 60 projections were determined and each projection sampled at 61 points. The tomograms were constructed on a $60 \times 60$ array of points by the method in Section 2 . The results are shown in Figs. 15a-e. Comparison of Figs. 15a-d with Fig. 5b clearly demonstrates that, for the reconstruction algorithm used, beam divergence up to $15^{\circ}$ can be ignored. 


\section{EXPERIMENTAL RESULTS}

A test phantom is shown in Fig. 16. It consists of a $2 \mathrm{~cm} x$ $5 \mathrm{~cm} \times 7 \mathrm{~cm}$ lucite block taped to a one millimeter aluminum sheet. There are two holes in the lucite block, each of diameter $1 \mathrm{~cm}$. The holes were filled with a $1.5 \%$ (weight/unit volume) solution of Hypaque. The contrast between the solutions and the surrounding lucite is provided by the $K$ absorption edge of iodine. The phantom was mounted on a protractor which revolved about its center. The center of the block was also the center of rotation. This assembly was then placed midway between the $x$-ray target and the input screen of the flouroscopic system. The tube was set at approximately $60 \mathrm{kVp}$ and the effective energy determined from absorption in aluminum was $22.8 \mathrm{keV}$. The tube current was approximately $1 \mathrm{~mA}$. The beam divergence angle was estimated to be $10^{\circ}$. Sixty projections at $3^{\circ}$ intervals were taken and each projection digitized and spatially and temporally averaged, as discussed in Section 3. $x$-ray field inhomogeities were removed from the data by first recording a blank projection (i.e., measuring the $x$-ray intensities without any object between the source and the image intensifier) at low tube current to prevent the saturation, computing the angular dependence of radiation and then correcting the projection data accordingly.

Figs. 17a-c show the tomograms obtained for this phantom. Fig. $16 a$ is obtained when the air-transmission value is set equal to the largest value at points outside the geometric shadow cast by the test phantom on the flourescent screen of the image-intensifier tube. It is clear from the theory and the computer simulation presented in 
Section 4 that the ring artifact in Fig. 17 a is due to the errors in the air-transmission value. The correct value of the air-transmission intensity can only be arrived at by trial and error, keeping in mind the fact that the correct value should make the ring in Fig. 17a disappear. Fig. 17b is the tomogram obtained from the same data as that used for Fig. 17a, except that the airtransmission value used has been corrected. By gray level slicing a tomogram one can display separately regions of different densities.

The above experiment with a slight change was repeated with the test phantom replaced by a dead rat. Instead of rotating the platform in Fig. 6, the $x$-ray tube and the image intensifier were rotated around the rat, thus representing more nearly the clinical situation. A total of 180 equaliy spaced projections were taken through part of the thorax containing the heart. The approximate location of the section for which the tomogram was made is shown in Fig. 18a. Fig. 18b shows the constructed tomogram with the air-transmission value set equal to the data obtained from rays outside the geometric shadow cast by the rat body. The ring artifact resulting from the errors in the air-transmission value is again evident. Fig. 18c results when the air-transmission value is corrected. Fig. 18d identifies the various anatomical features for the tomogram. 


\section{REFERENCES}

1. N. A. Baily and R. L. Crepeau, "Capabilities of a Single Scan TV-Radiographic System for Digital Data Acquistion," Investigative Radiology, Vol. 6, pp. 273-279, 1971.

2. N. A. Baily and R. L. Crepeau, "Performance of a Large Screen "Flouroscopic Imaging System," Proc. Photo-Optical Instrumenttàtion Eng., Vol. 43, pp. 135-139, 1973.

3. N. A. Baily and R. L. Crepeau, "The Capability of Flouroscopic Systems to Determine Differential Roentgen-ray Absorption," Radiol., Vol. 115, pp. 439-445, 1975.

4. M. V. Berry and D. F. Gibbs, "The Interpretation of 0ptical Projection," Proc. Roy. Soc. London, Ser. A, Vol. 314, pp. 143-152, 1970.

5. R. N. Bracewell and A. C. Riddle, "Inversion of Fan-Beam Scans in Radio Astronomy," The Astrophys. J., Vol. 314, pp. 143-152, 1970.

6. S. K. Chang and C. K. Chow, "The Reconstruction of Three-Dimensional Objects From Two Orthogonal Projections and its Applications to Cardiac Cieneagiography," IEEE Trans. Comput., Vol. C-22, pp. 18-28, Jan. 1973.

7. A. M. Cormack, "Representation of a Function by its Line Integrals with Some Radiological Applications-11," J.Appl. Phys., Vol. 35, pp. 2722-2727, 1963.

8. A. M. Cormack, "Representation of a Function by its Line Integral with Some Radiological Applications-11, J.Appl. Phys., Vol. 35, pp. 2908-2913, 1964.

9. A. M. Cormack, "Reconstruction of Densities from Their Projections with Applications in Radiological Physics, Phys. Med. Biol., Vol. 18, pp. 195-207, 1973.

10. R. L. Crepeau, N. A. Baily, and N. R. Silverman, "Applications of aDigitized Radiographic-Flourographic Processing System to Physiologic Data Extraction," Proc. San Diego Biomedical Symposium, Vol. 11, pp. 317-32 1, 1972.

11. R. A. Crowther, "Procedures for Three-Dimensional Reconstruction of Spherical Viruses by Fourier Synthes is from Electron Micrographs," Phil. Trans. Roy. London, Ser. B, Vol. 26I, Pp. 221-230, 1971.

12. R. A. Crowther and L. Amos, Harmonic Analys is of Electron Microscope Images with Rotational Symmetry," J. Mol. Biol., Vol. 60, pp. 123-130, 1971. 
13. R.'A. Crowther, D. J. DeRosier, and A. Klug, "The Reconstruction of a Three-Dimensional Structure from Projections and its Applications to Electron Microscopy," Proc. Roy. Soc. Lond., A317, pp. 319-340, 1970.

14. D. J. DeRosier and P. B. Moore, "Reconstruction of ThreeDimensional Images from Electron Micrographs of Structures with Helical Symmetry," J. Mol. Biol., Vol. 52, pp. 355-369, 1970.

15. D. J. DeRosier and A. Klug, "Reconstruction of Three-Dimensional Structures from Electron Micrographs," Nature, Vol. 217, Pp. 130134, 1968.

16. P. Gilbert, "Iterative Methods for the Reconstruction of ThreeDimensional Objects from Projections," J. Theor. Biol., Vol. 36, pp. 105-117, 1972 .

17. R. Gordon, R. Bender, and G. T. Herman, "Algebraic Reconstruction Techniques (ART) for Three-Dimensional Electron Microscopy and X-ray Photography," J. Theor. Biol., Vol. 29, pp. 471-48i, 1971 .

18. R. Gordon and G. T. Herman, "Reconstruction of Pictures from Their Projections," Comm. A.C.M., Vol. 14, pp. 759-768, 1971.

19. D. G. Grant, "TOMOSYNTHESIS: A Three-Dimensional Radiographic Imaging Techniques," IEEE Trans. Biomed. Eng., Vol. BME-19, pp. 20-28, Jan. 1972 .

20. G. T. Herman, "Two Direct Methods for Reconstructing Picutares from Their Projections: A Comparative Study," Computer Graphics and Image Processing, Vol. 1. pp. 123-144, 1973.

21. G. T. Herman, A. Lent, and S. Rowland, "ART: Mathematics and Applications. A report on the mathematical foundations and on the applicability to real data of the Alyegraic Reconstruction Techniques," J. Theor. Biol., Vol. 42, pp. 1-32, 1973.

22. G. T. Herman and S. Rowland, "Resolution in ART: an Experimental Investigation of the Resolving Power of an Albebraic Picture Reconstruction Techniques," J. Theor. Biol., Vol. 33, Pp. 213-223, 1971 .

23. G. N. Houncefield "A Method of and Apparatus for Examination of a Body by Radiation Such as $x$ or gamma Radiation," Patent Specification 1283915, Patent Office, London, 1972.

24. A. C. Kak, "Algorithms for Reconstruction," To appear in Proceeding of the International Conference on Cardiovascular Imaging and Image Processing, Stanford University, July 1975.

25. D. E. Kuhl and R. Q. Edwards, "Reorganizing Data from Transverse Section Scans of the Brain Using Digital Processing," Radiology, Vol. 91, pp. 975-983, 1968. 
26. R. M. Mersereau, "Recovering Multi-Dimensional Signals from Their Projections," Comp. Graph. Image Proc., Vol. 1, No. 6, pp. 179-195, Oct. 1973.

27. R. M. Merseréau and A. V. Oppenheim, "Digital Reconstruction of Multidimensional Signals from Their Projections," Proc. IEEE, Vol. 62, pp. 1319-1338, 1974.

28. T. M. Peters, P. R. Smith, and R. D. Gibson, "Computer Aided Transverse Body-Section Radiography," Brit. J. Radia, Vol. 46, pp. 314-317, 1973.

29. G. N. Ramachandran and A. V. Lakshminarayanan, "Three-Dimensional Reconstruction from Radiographs and Electron Micrographs: 111. Description and application of the convolution method." Indian J. Pure Appl. Phys., Vol. 9, pp. 997-1003, 1971.

30. G. N. Ramachandran and A. V. Lakshminarayanan, "Three-Dimensional Reconstruction firom Radiographs and Electron Micrographs: 111. Application of convolutions instead of Fourier transforms," Proc. Nat. Acad. Sci., Vol. 68, No. 9, pp. 22362240 .

31. R. A. Robb, J. F. Greenleaf, E. L. Ritman, S. A. Johnson, J. D. Sjostrand, G. T. Herman, and E. H. Woods, "Three Dimensional Visualization of the Intact Thorax and Contents: A Technique for Cross-Sectional Reconstruction from Multiplanar X-ray Views," Computers and Biomedical Research, Vol. 7, pp. 395$419,1974$.

32. A. Rosenfeld and A. C. Kak, Digital Picture Processing, Academic Press, New York, Fal1 1975. (In print)

33. L. A. Shepp and B. F. Logan, "The Fourier Reconstruction of a Head Section," IEEE Trans. Nucl. Sci., Vol. NS-21, pp. 21-43, June 1974.

34. M. M. Ter-Pogossian, The Physical Aspects of Diagnostic Radiology, Harper and Row Publishers, New York, 1967.

35. 0. Tretiak, D. Ozonoff, J. Klopping, and M. Eden, "Calculation of Internal Structure from Multiple Radiograms," in Proc. Two-Dimensional Digital Signal Processing Conf., Univ. of Missouri-Columbia, pp. $6-2-1-6-2-3$.

36. E. C. McCullough, H. L. Baker, Jr., O. W. Houser, and D. F. Reese, "An Evaluation of the Quantitative and Radiation Features of a Scanning X-ray Transverse Axial Tomograph: The EMI Scanner;": Radiation Physics, Vol. 111, pp. 709-715, June 1974. 
APPENDIX A

DERIVATION OF EXPRESSION FOR PROJECTION

DATA USED IN COMPUTER SIMULATIONS

In this appendix we show how an analytic expression for the projection data, $P_{\theta}(t)$, for a certain class of picture functions may be derived. This data can easily be generated and subsequently used in the reconstruction algorithm in order to test the various effects addressed in this paper.

Now it should be noted here that it is fairly easy to obtain a sort of "pseudo-projection data" for any digital picture [21]. In order to do so, one can think of laying down "strips" centered around each line $L(t, \theta)$ in a given projection, as shown in Figure A1.1. Now if the picture to be reconstructed assumes a constant value $f_{i j}$ in each square centered at $(i, j)$, one can estimate $P_{\theta}(t)$ by summing $f_{i j}$ for those block centers $(i, j)$ which are included in the proper strip. This type of data, however, does not necessarily closely approximate the actual $P_{\theta}(t)$ and hence is not generally very useful for testing the reconstruction algorithms. Now one could easily argue that this procedure could be made exact by simply taking the actual fraction of each square included by a given ray into account, rather than by simply summing the $f_{i j}$ for the block centers $(i, j)$ included. Although this can be done, it is a time consuming procedure to compute the appropriate fractions for each block for each ray of each projection. What is desired is an analytic expression for $P_{\theta}(t)$ for some class of pictures, so that exact test data could be rapidly generated. 
Now as it turns out, the line integrals $P_{\theta}(t)$ can be worked out precisely without difficulty if the picture function $f(x, y)$ is such that it assumes a constant values, say $c_{1}$, inside of an ellipse and is zero elsewhere. This was suggested in a recent paper by Shepp and Logan [33]. It will then be easy to obtain the projection data for any picture which is a superposition of such ellipses. To make things more precise, consider first an ellipse centered at the origin with semi-major and semi-minor axes given by $A$ and $B$, respectively:

$$
\frac{x^{2}}{A^{2}}+\frac{y^{2}}{B^{2}}=1
$$

Now define $f(x, y)$ such that:

$$
\begin{aligned}
& f(x, y)=C_{1} \text { for } \frac{x^{2}}{A^{2}}+\frac{y^{2}}{B^{2}} \leq 1 \\
& f(x, y)=0, \text { otherwise }
\end{aligned}
$$

In order to obtain the projection function $P_{\theta}(t)$ for $f(x, y)$, we need to compute:

$$
P_{\theta}(t)=\delta_{L(t, \theta)} f d s
$$

for each line $L(t, \theta)$ in the $\theta$-projection, as shown in Figure Al.2. [Note also that we further arbitrarily restrict the size of the ellipse to be such that it is contained entirely within the unit circle centered at the origin. In this way, all of our simulated pictures will be such that $f(x, y)=0$ for $x^{2}+y^{2}>1$. This, in turn, requires that $P_{\theta}(t)$ be zero for all $|t|>1$, for all $\theta_{\text {.] }}$ 
Since $f(x, y)=C_{1}$ for all points inside the ellipse, it is clear that for a given line $L(\tau, \theta)$ the value of $P_{\theta}(\tau)=\int_{L}(\tau, \theta)$ ds is given simply by $C_{1}$ times the length, $\overline{Q_{1} Q_{2}}$, of the line segment, $Q_{1} Q_{2}$ (see Figure Al.2). If $\left(x_{1}, y_{1}\right)$ and $\left(x_{2}, y_{2}\right)$ are the coordinates of points $Q_{1}$ and $Q_{2}$, respectively, then it may be seen from

Figure Al. 1 that

$$
\overline{Q_{1} Q_{2}}=\frac{\left|x_{2}-x_{1}\right|}{\sin \theta}
$$

So we need to compute the points of intersection, $Q_{1}, Q_{2}$, of the line $L(\tau, \theta)$ with the ellipse.

Now the 1 ine $L(\tau, \theta)$ is given by:

$$
x \cos \theta+y \sin \theta=\tau
$$

Substituting the value of $y$ as given by (A3) in (A1) and calculating the roots of the resulting equation, we get

$$
x_{1}, x_{2}=\frac{2 A B \sin \theta}{\left(A^{2} \cos ^{2} \theta+B^{2} \sin ^{2} \theta\right)}\left[\cdot \pm \sqrt{\left(A^{2} \cos \theta+B^{2} \sin ^{2} \theta-\tau^{2}\right)}\right]
$$


Now (A4) implies that the value of $\tau$ which forces the radical to be zero must be the value of $\tau$ for which $x_{1}=x_{2}$, and hence for which $\left|x_{1}-x_{2}\right|=0$. This is precisely the situation for which $L(\tau, \theta)$ is just tangent to the ellipse, and so this value of $\tau$ is the "projection half-width". This is denoted by $a=a(\theta)$, and is illustrated in Figure Al.2. Clearly, we have:

$$
a(\theta)=\sqrt{A^{2} \cos ^{2} \theta+B^{2} \sin ^{2} \theta}
$$

So we now obtain:

$$
\left|x_{1}-x_{2}\right|=\frac{2 A B \sin \theta}{a^{2}} \sqrt{a^{2}-t^{2}}
$$

and substituting into (A2) yields:

$$
P_{\theta}(t)=\frac{2 C_{1} A B}{a^{2}} \sqrt{a^{2}-t^{2}}
$$

As a special check of the result (A7), it is clear that $P_{\theta}(t)$ should assume a value of $2 C_{1} B$ for the case $\theta=0, t=0$. This is evident from Figure Al.2.

In order to obtain $P_{\theta}(t)$ when the ellipse described above is centered at $(h, k)$ and is rotated through angle $\alpha$, as depicted in Figure Al.3, only a slight modification is needed.

It is not difficult to show that the projection data for this new picture function is related to $P(t, \theta)$ of $(A 7)$ by:

$$
P^{\prime}(t, \theta)=P[t-s \cos (\gamma-\theta), \theta-\alpha]
$$

where,

$$
s=\sqrt{h^{2}+k^{2}} \text { and } \gamma=\tan ^{-1}\left(\frac{k}{h}\right)
$$


That is, $(s, \gamma)$ are the polar coordinates of the translated center, $(h, k)$. Finally, if a picture function $f_{2}(x, y)$ is formed by superimposing $\mathrm{N}$ single ellipse pictures, where each ellipse assumes some constant value $c_{i}$ inside and is zero outside, it is clear that (All) may be modified to write the projection data for $f_{2}(x, y)$ as:

$$
P_{f_{2}}(t, \theta)=\sum_{i=1}^{N} c_{i} \cdot P_{i}\left[t-s_{i} \cos \left(\gamma_{i}-\theta\right), \theta-\alpha_{i}\right)
$$

where $s_{i}, \gamma_{i}$, and $\alpha_{i}$ describe the position and orientation of each separate ellipse in the picture plane.

For the case of fan beam ( $i . e .$, diverging beam) geometry, the same procedure may be followed, except that now the distance to be calculated in each case is as shown in Figure A1.4.

Finally, it should be noted that no $x$-ray tube in practice has a zero spot size and no beam, however, collimated, will, have zero "thickness". It is, therefore, desireable to be able to simulate data that would result from "rays" of some thickness, as opposed to the ideal "pencil beams" considered thus far. To this end, consider the situation shown in Figure A1.5. Here it is assumed that the detectors measure the average value of the line integral of $f(x, y)$ over the range of values, $t_{1}-T / 2<t<t_{1}+T / 2$, where $T$ is the "strip width". This, of course, is equivalent to finding the area of a given strip and dividing it by $T$. For example, the projection data for this strip integral case may be found simply from A7 for the single ellipse $x^{2} / A^{2}+y^{2} / B^{2}=1$ as: 


$$
\begin{aligned}
& P_{\text {strip }}(t, \theta)=\frac{1}{T} \int_{t-T / 2}^{t+T / 2} P(\lambda, \theta) d \lambda=\frac{2 A B C}{a^{2} T} \int_{t-T / 2}^{t+T / 2} \sqrt{a^{2}-\lambda^{2}} d \lambda= \\
& \frac{A B C_{1}}{a^{2} T}\left[\lambda \sqrt{a^{2}-\lambda^{2}}+a^{2} \sin ^{-1} \frac{\lambda}{a}\right] \mid \begin{array}{l}
t+T / 2 \\
t-T / 2
\end{array}
\end{aligned}
$$

Now the projection data for the case of $\mathrm{N}$ ellipses with various positions and orientations can again be found by analogy to (A9). 


\section{FIGURE CAPTIONS}

1. A schematic diagram of the linear motion of the radiation sourcedetector combination required to generate each projection.

2. The schematic diagram shows that in a flouroscopic system an entire projection is taken at the same time.

3. The one dimensional function $P_{\theta}(t)$ is the projection of the function $f(x, y)$ in the direction of $\theta+90^{\circ}$.

4. This figure illustrates the back-projection of the filtered projection function $\mathrm{Q}_{\theta}(t)$.

5. a) The pattern used for computer simulation.

b) The image reconstructed from the projections of Fig. 5 a.

c) The relative gray levels in Fig. 5 a.

d) The relative gray levels in Fig. 5 b.

6. A block schematic of a system for computerized tomography imaging using flouroscopic images.

7. The figure shows the part of the TV frame that is actually digitized.

8. a) Absorbing material in a parallel beam of $x$-rays. This would be the case for linear-scan tomography.

b) $x$-ray beam in a flouroscopic system is diverging as shown here.

c) Absorbing material in a diverging beam.

d) For small angles $\theta$ in Fig. 8c the dependence on z-axis can be ignored and the resulting geometry is as shown here.

9. This figure shows that the digitized data is proportional to $\log 1$ rather than 1 , where 1 is the intensity of x-rays impinging on the flourescent screen. In our experiments we used a 7-bit quantizer (128 levels) and the projection data was typically contained in the range 30 to 120 . For this range of the quantizer the curve is 1 inear.

10. All the analog values between $z_{i}$ and $z_{i+1}$ are assigned the discrete value $q_{i}$ by the quantizer.

11. a) The d.c. shift in all the projections caused by the error in the air-transmission value after the projection have been corrected for scan-to-scan variations.

b) Derivative of the function is Fig. Ila. 
12. Computer simulation of the effect of air-transmission value errors. Note the ring artifact.

13. These reconstructions indirectly show the importance of spatial and temporal averaging in a flouroscopic system, since it doesn't pay to have more quantization levels than that decided by the signal-to-noise ratio in the projections. The figure shows the reconstructions with projection data quantized to (a) 8 levels, (b) 16 levels, (c) 32 levels, and (d) 128 levels.

14. Figure shows how the data from diverging radiation can be rearranged to approximately look like the data from parallel radiation.

15. These reconstructions show that the beam divergence effects are insignificant for divergence angles up to $15^{\circ}$. The reconstructions shown are for (a) $5^{\circ}$, (b) $10^{\circ},(c) 15^{\circ}$, (d) $20^{\circ}$, and (e) $60^{\circ}$.

16. (a) Lucite block with two holes that are filled with iodine solution.

(b) The block is then taped to a $1 \mathrm{~mm}$ thick aluminum backing and mounted between two aluminum blocks.

17. (a) Image of a tomogram through the lucite block. The ring is an artifact generated by errors in the air-transmission value.

(b) The tomogram after the air-transmission value is corrected.

18. (a) The approximate location of the thoracic section for which the tomogram was made.

(b) The tomogram with no correction for the air-transmission value.

(c) .Tomogram with the air-transmission value corrected.

(d) Identification of feature in Fig. $18 \mathrm{c}$. 


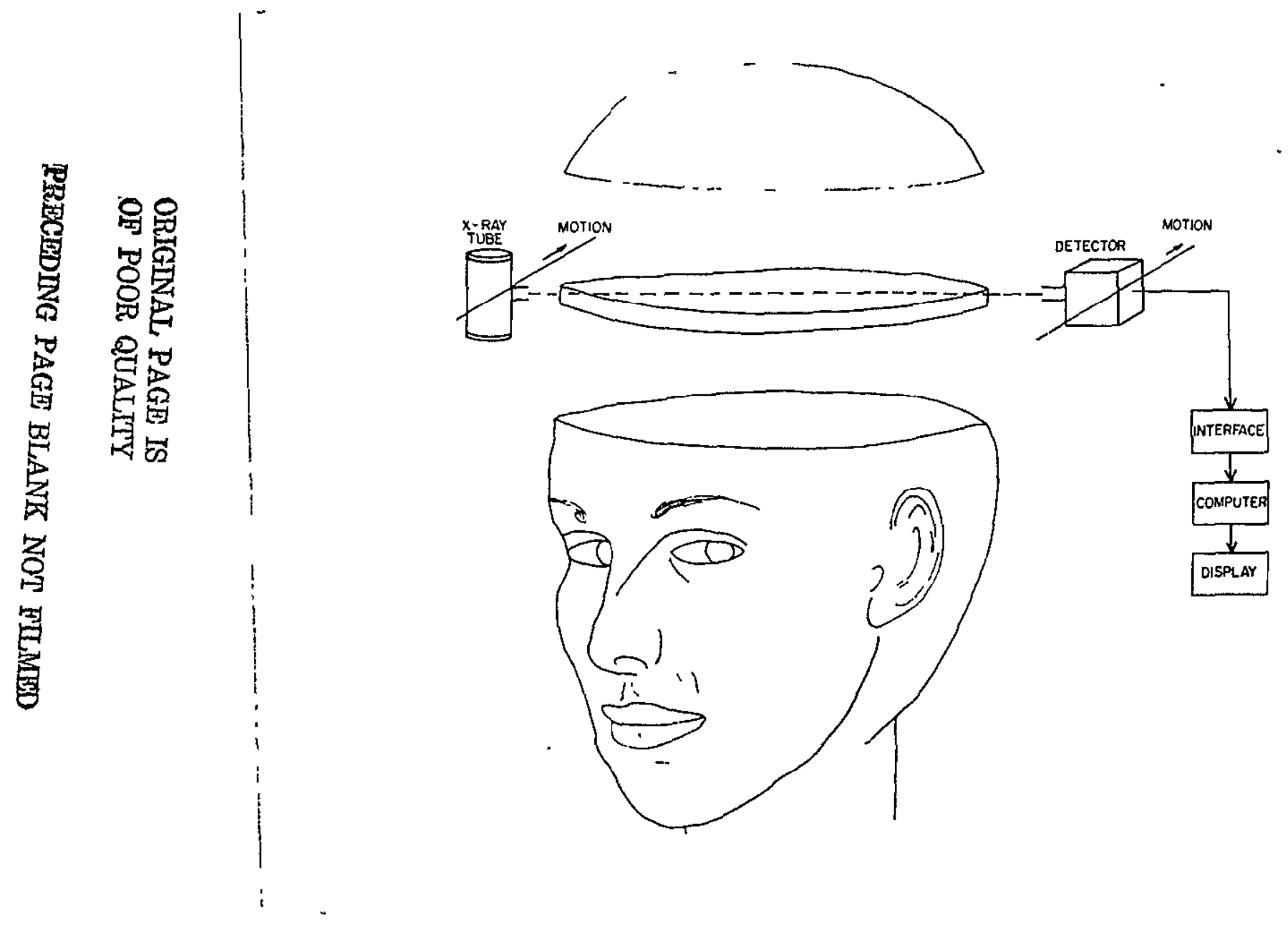

FIG. 1 


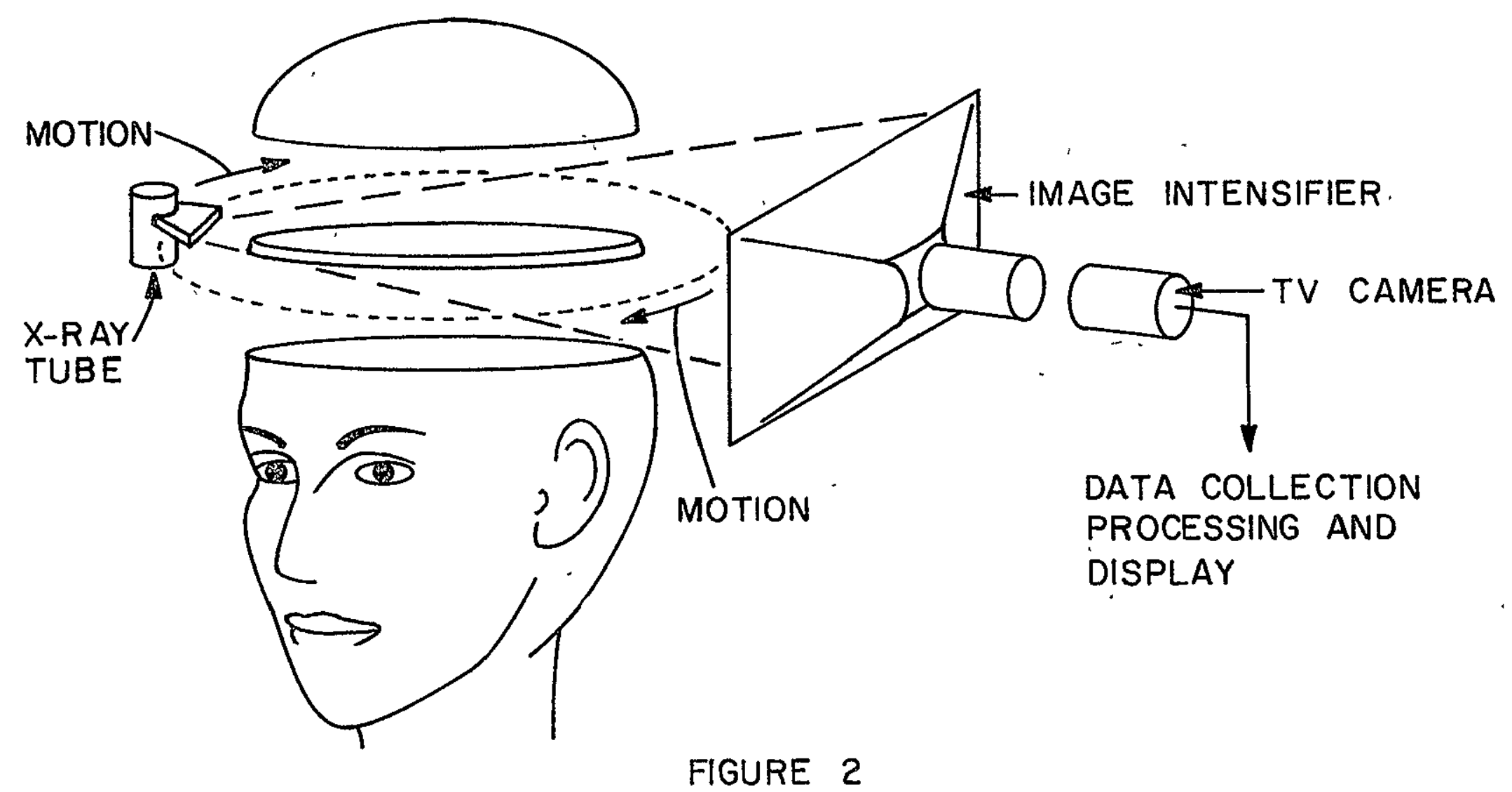




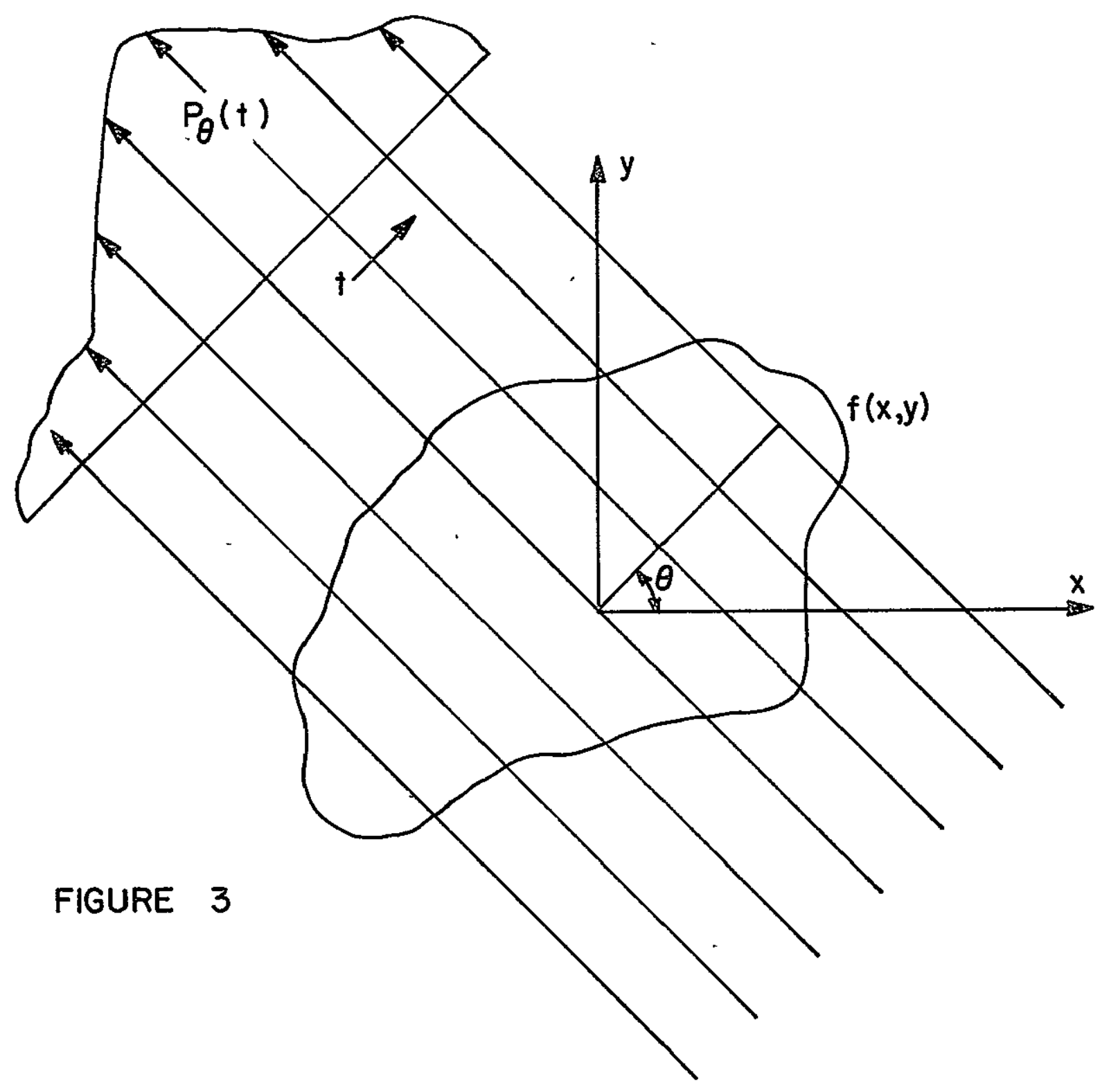




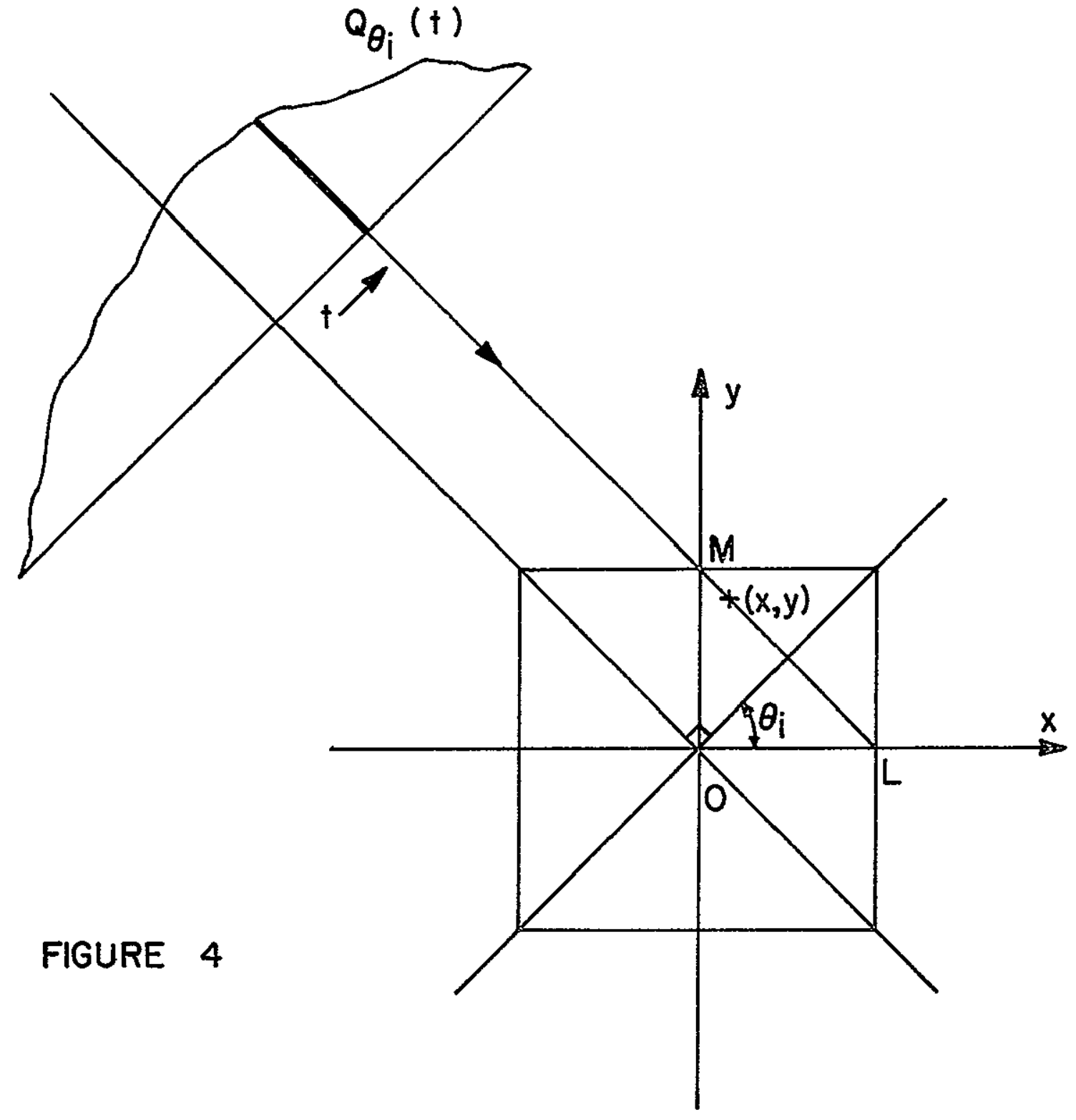




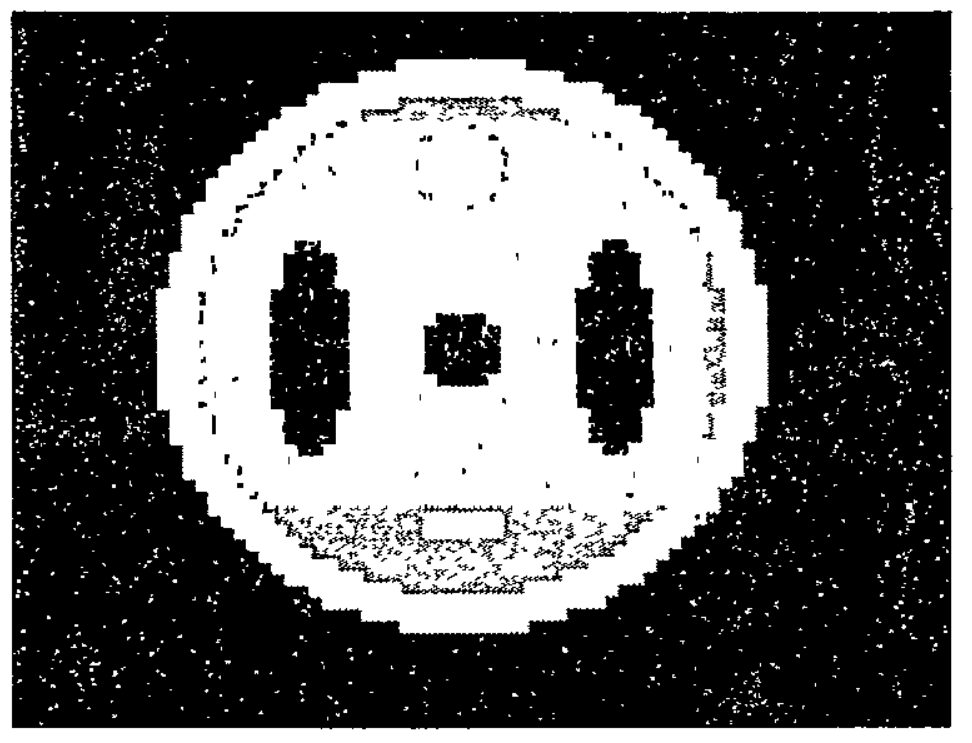

Flgure $5 \mathrm{a}$

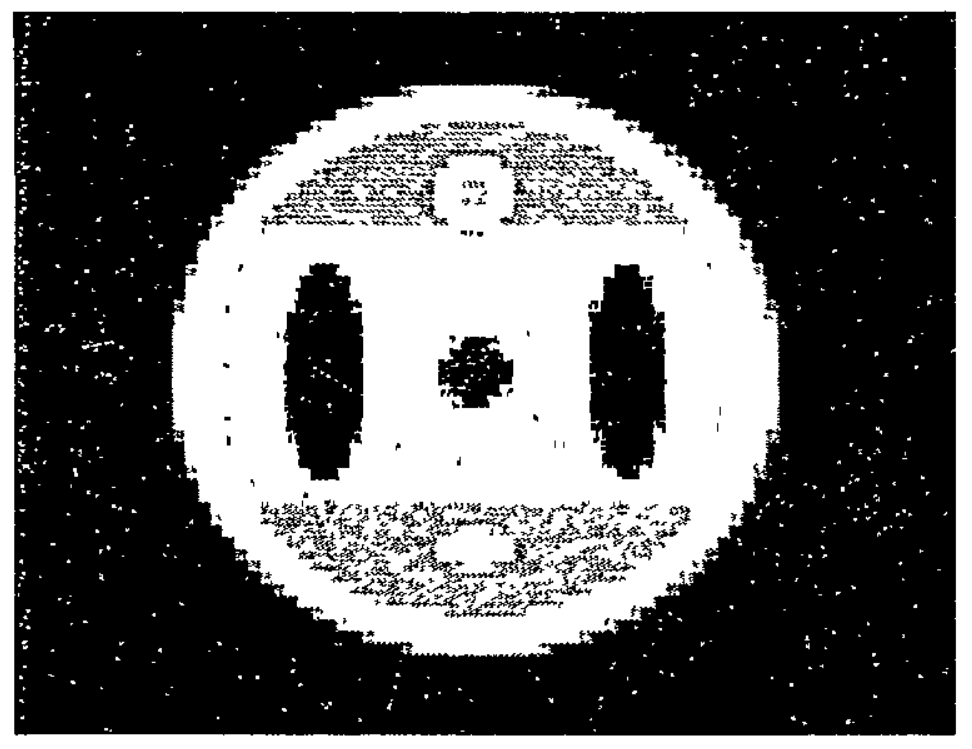

Fisure $5 b$ 


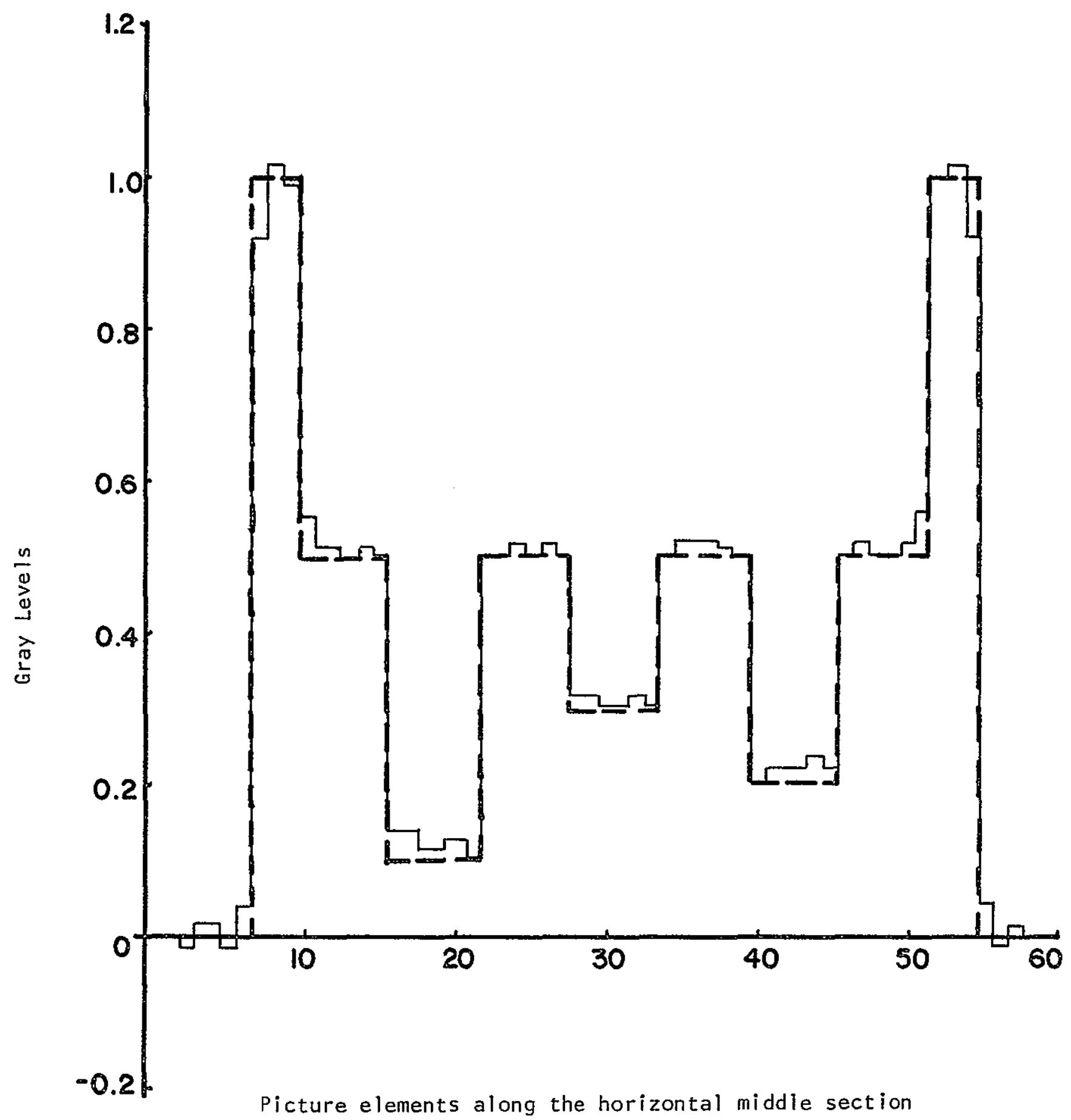

Fig. $5 \mathrm{c}$ 


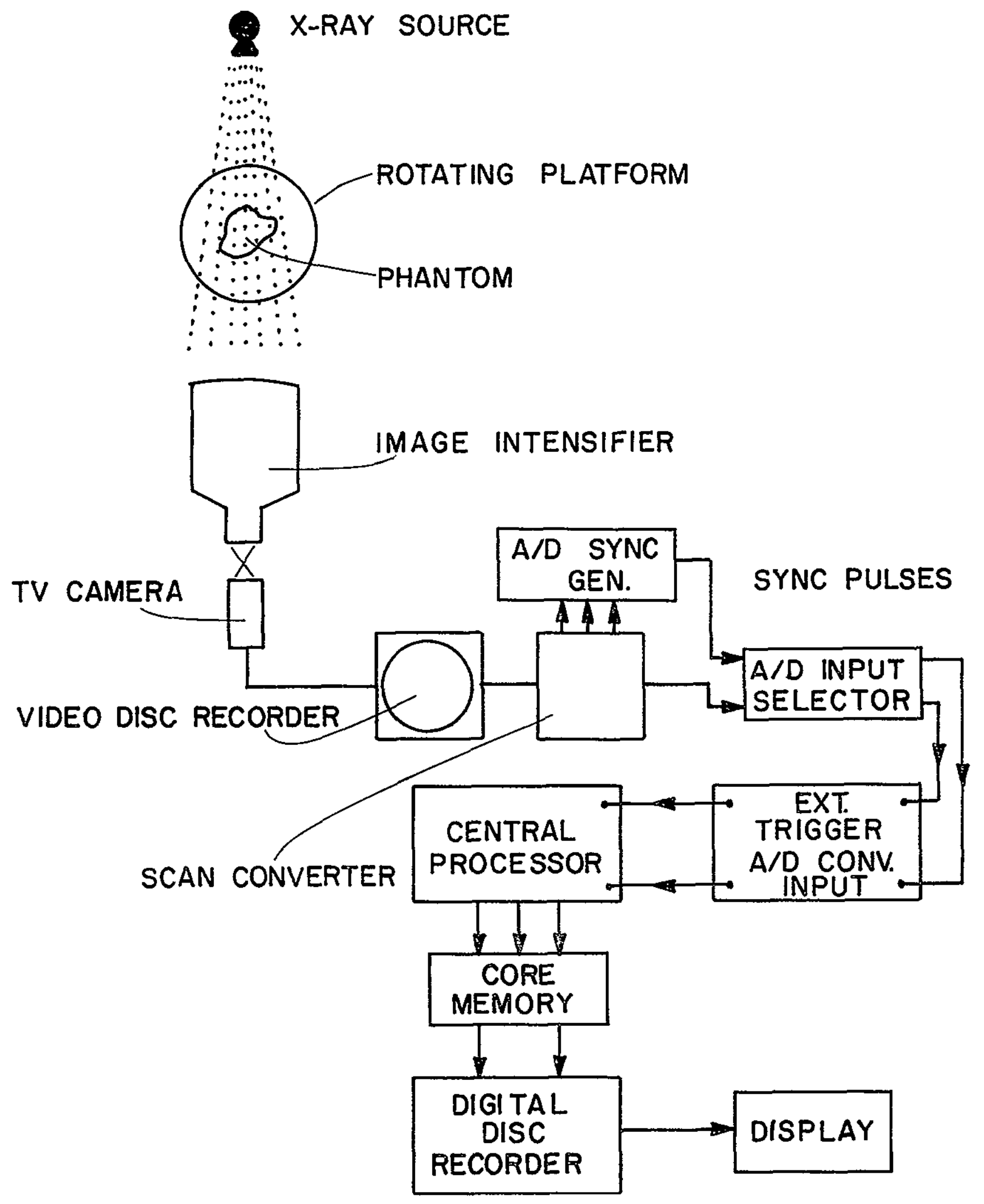

FIGURE 6 


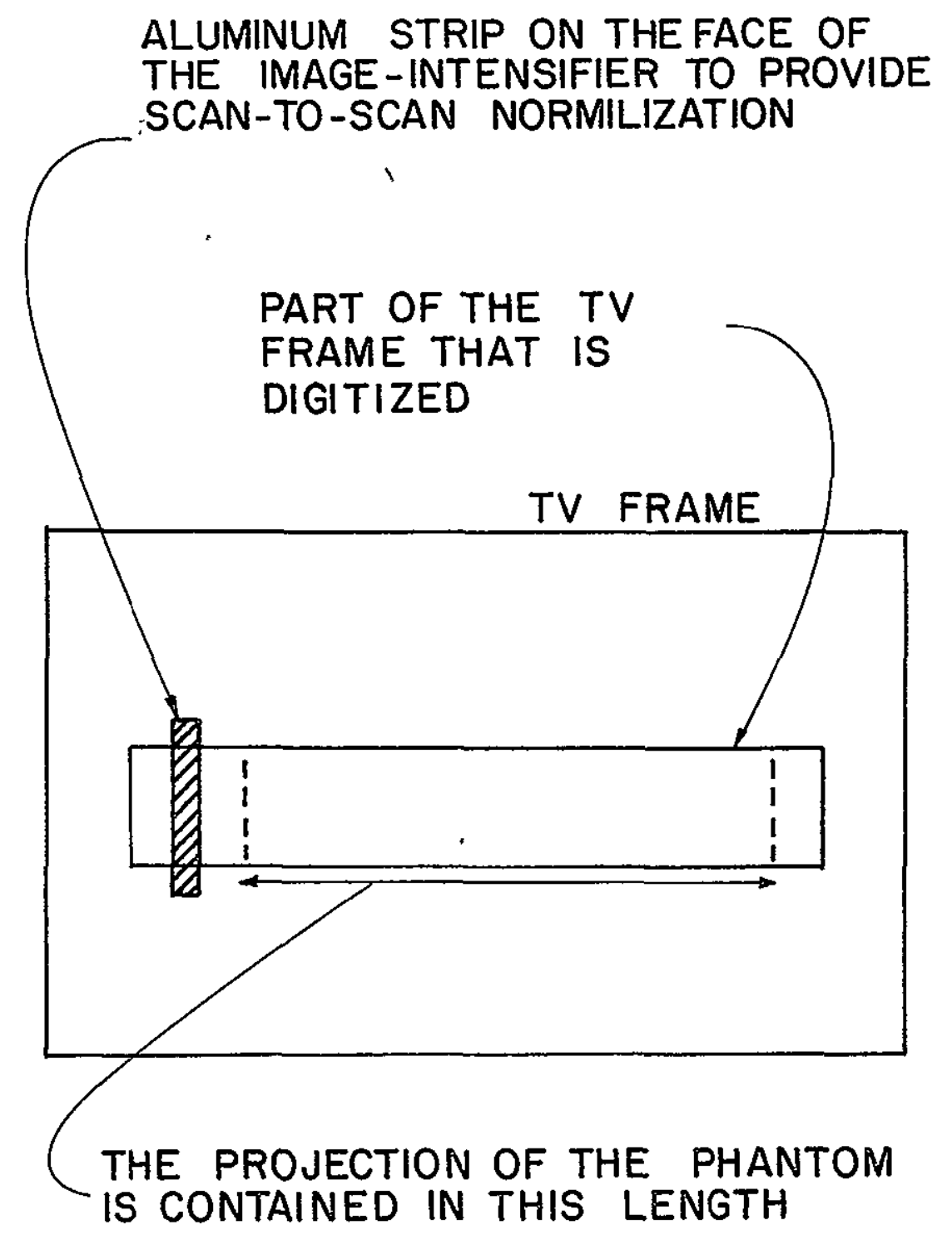

FIGURE 7 


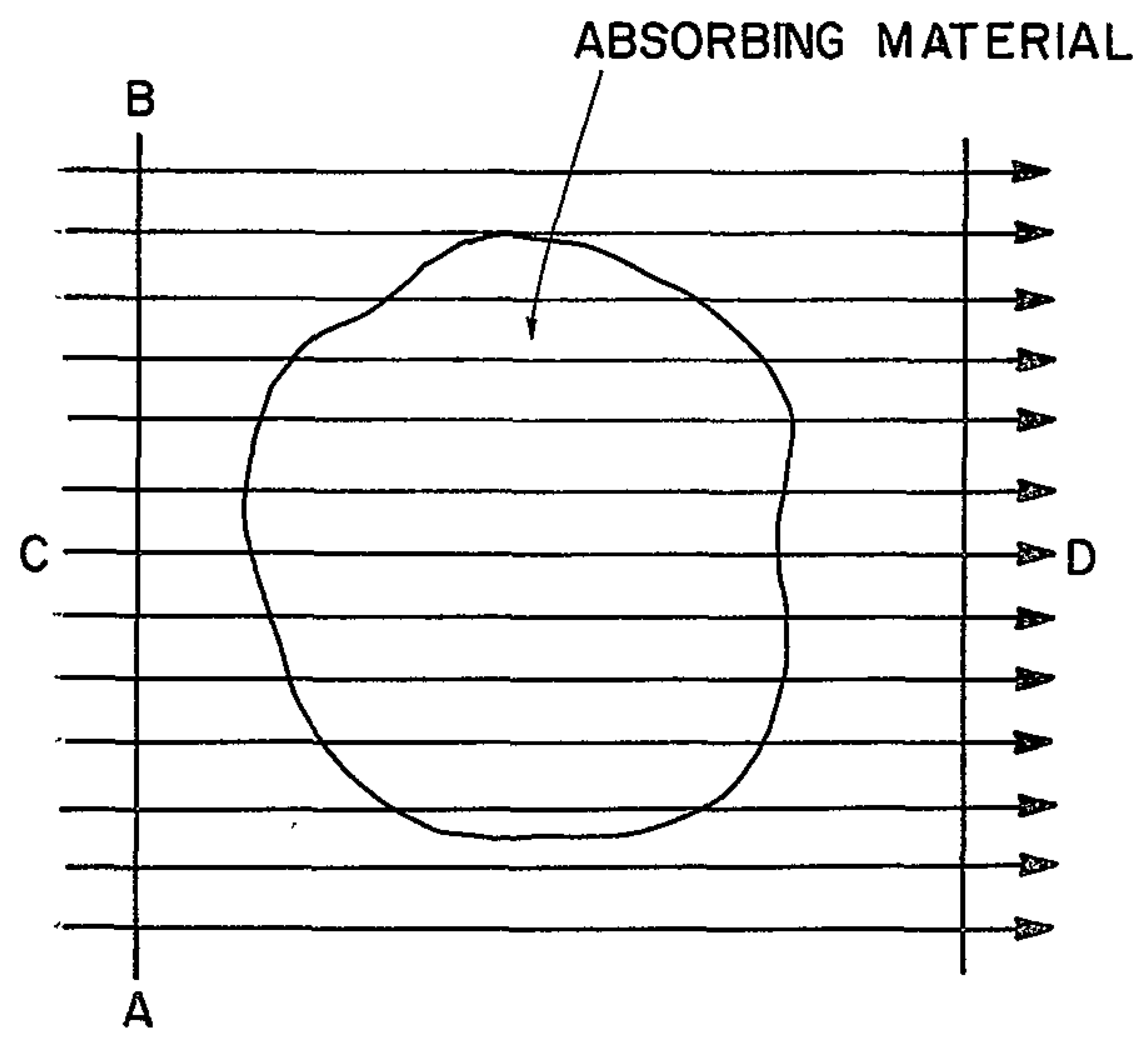

FIGURE 8a

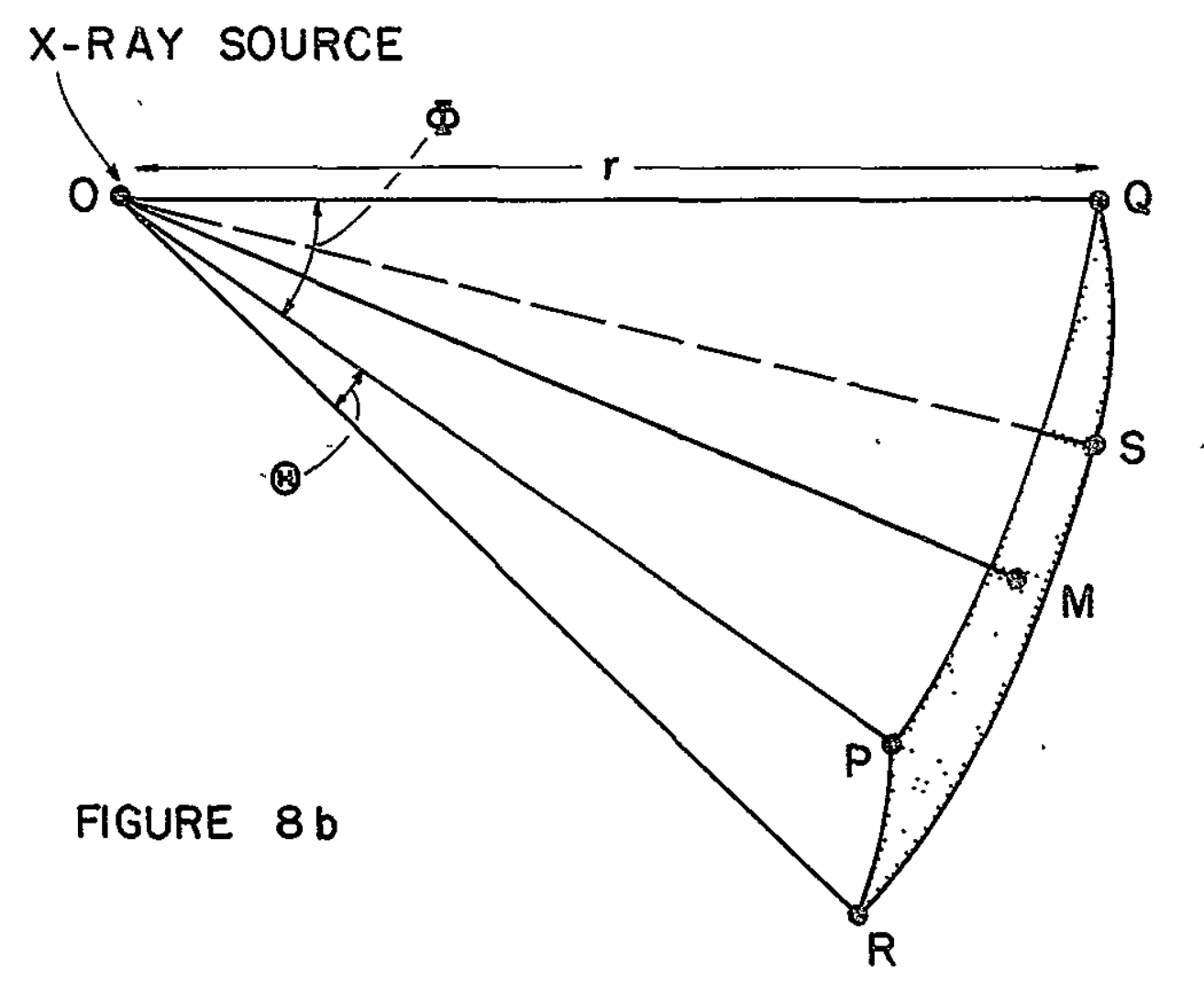



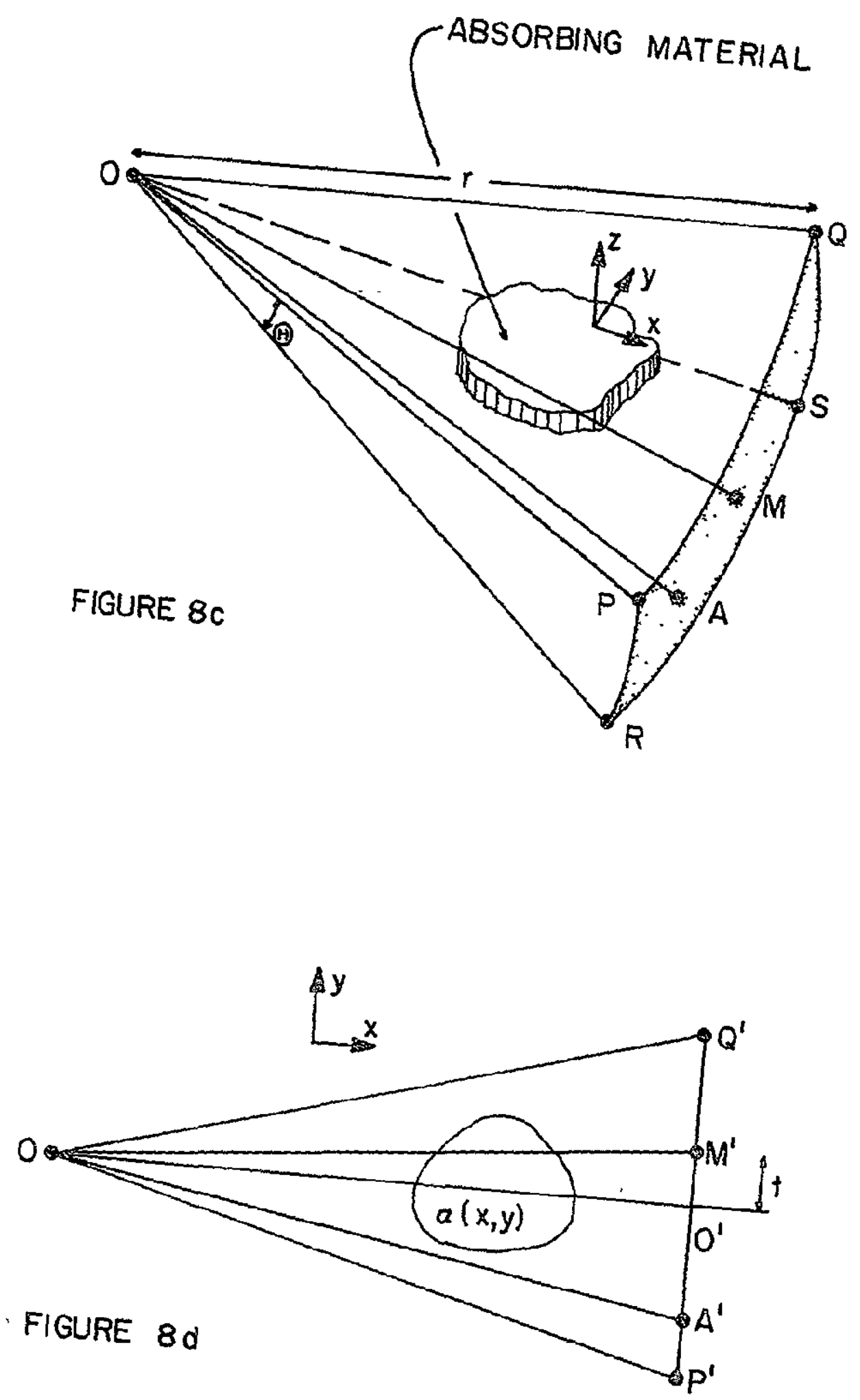


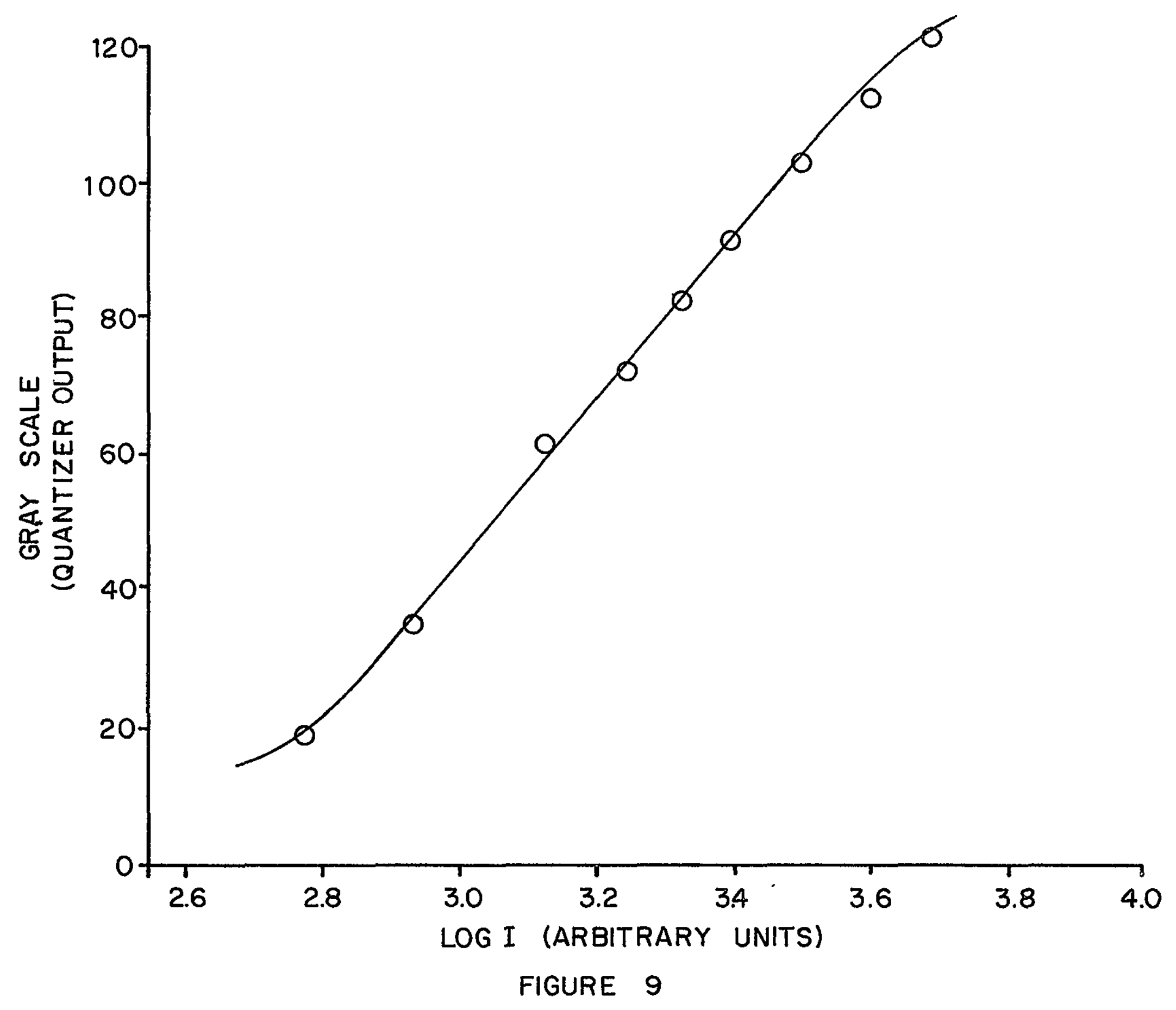




\section{SAMPLE VALUE RANGE}

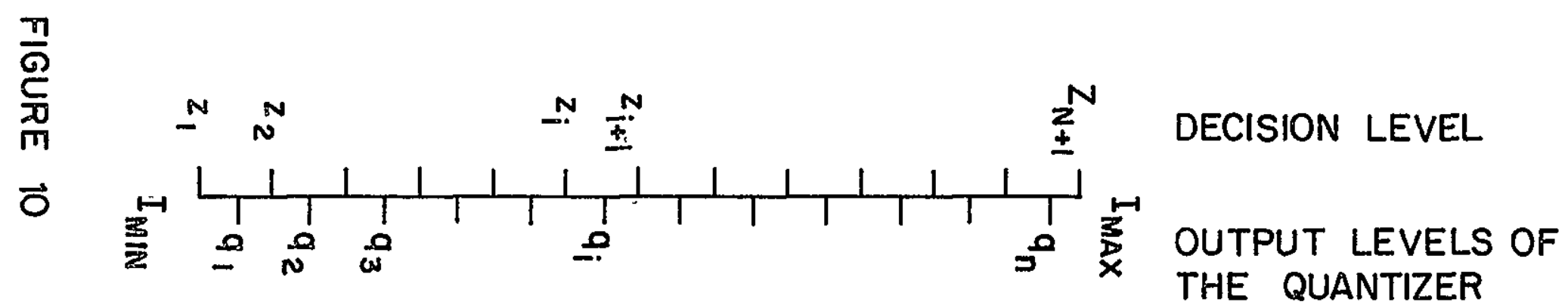



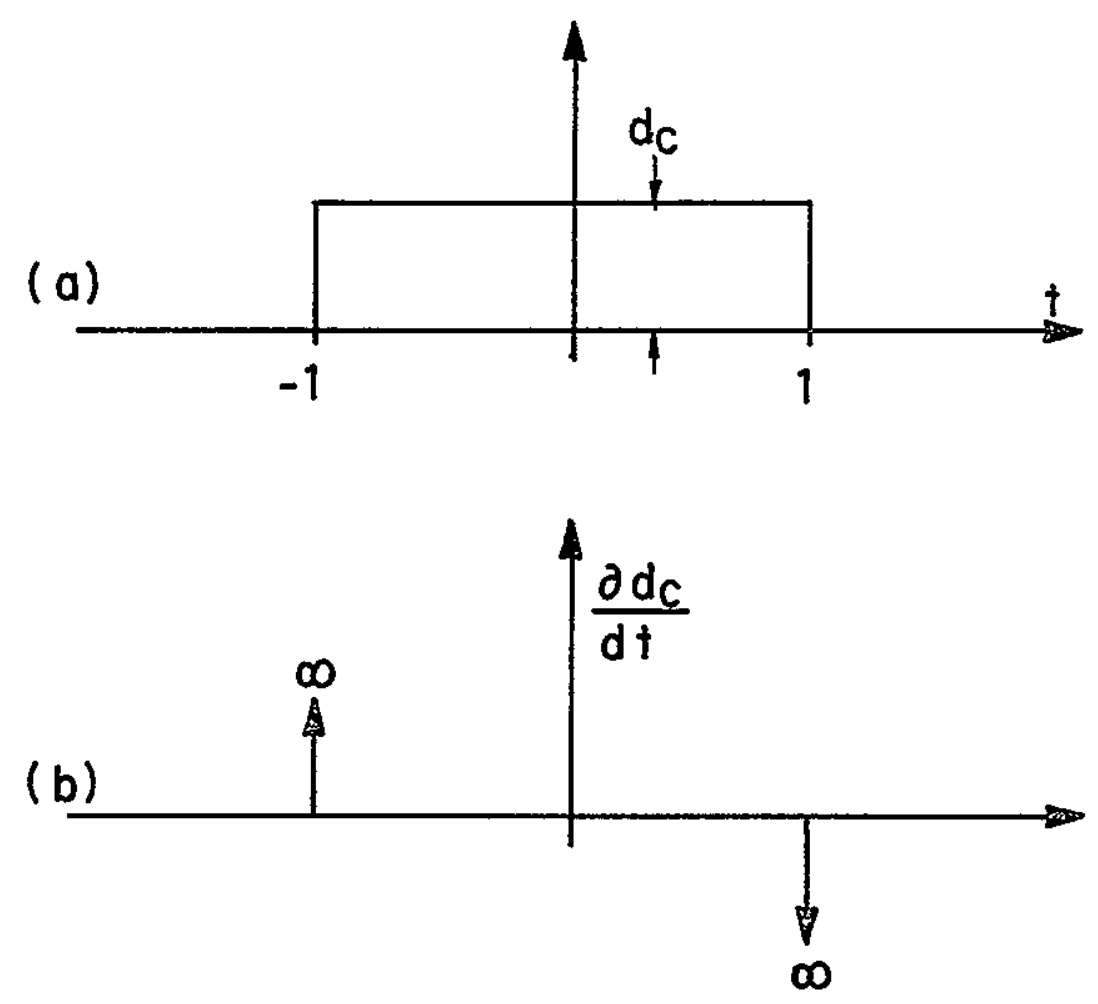

FIGURE 11 


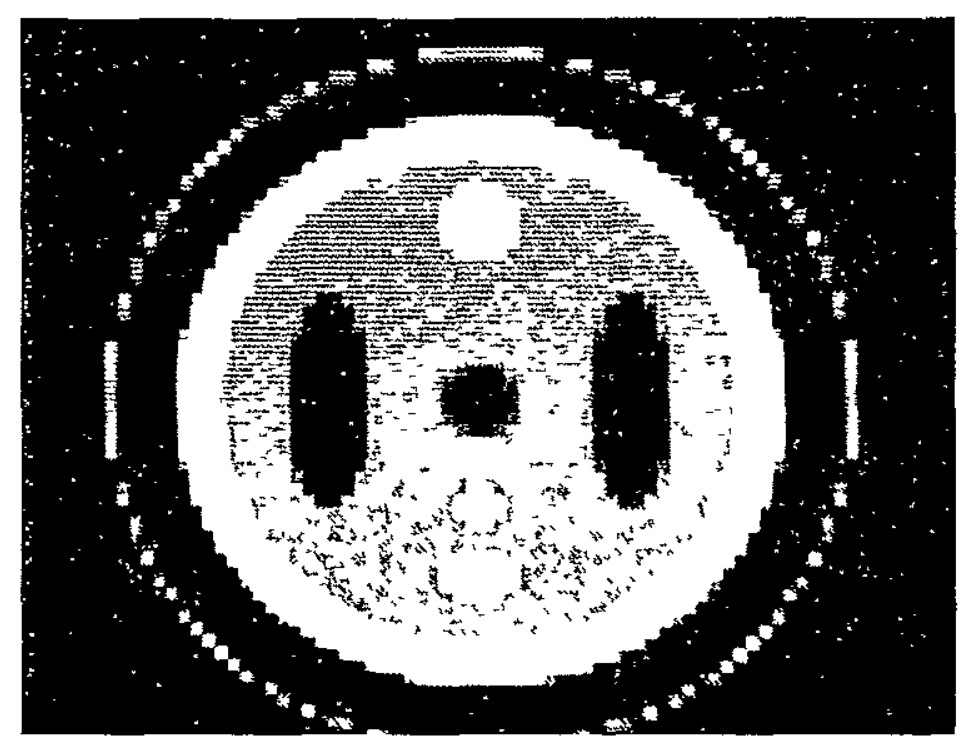

Flgure 12 


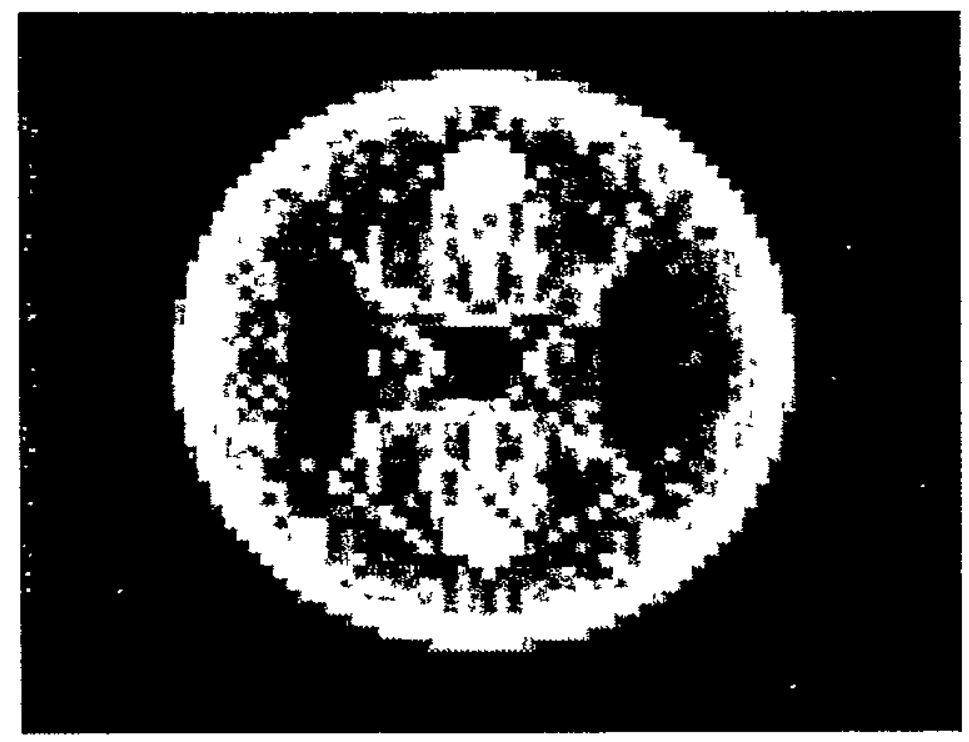

Flguxe 13a

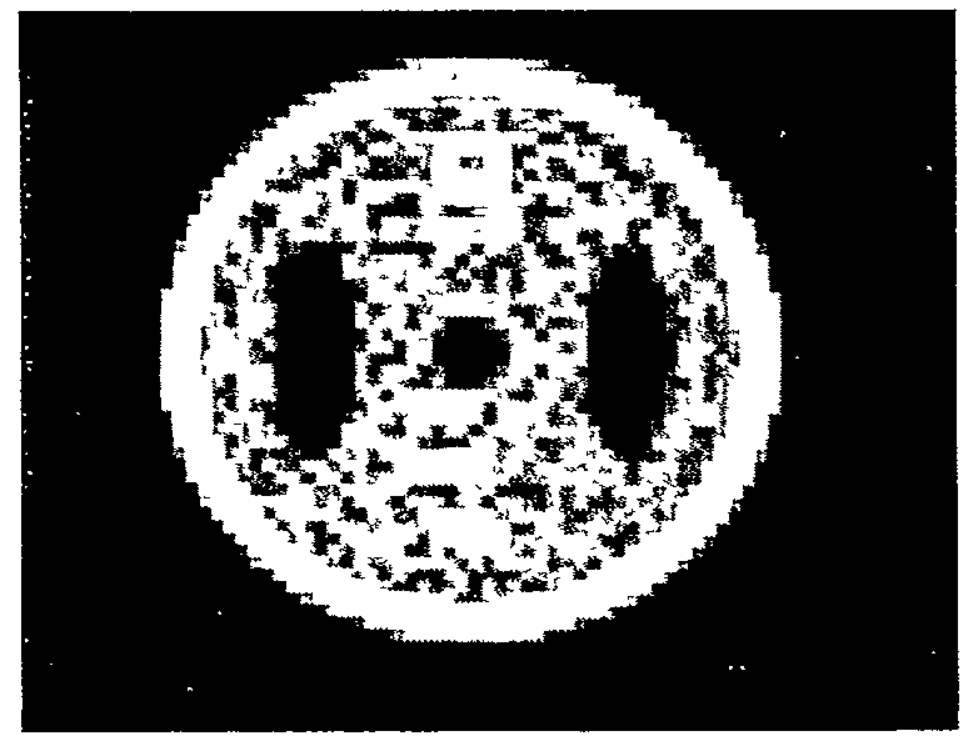

Figure 13b 


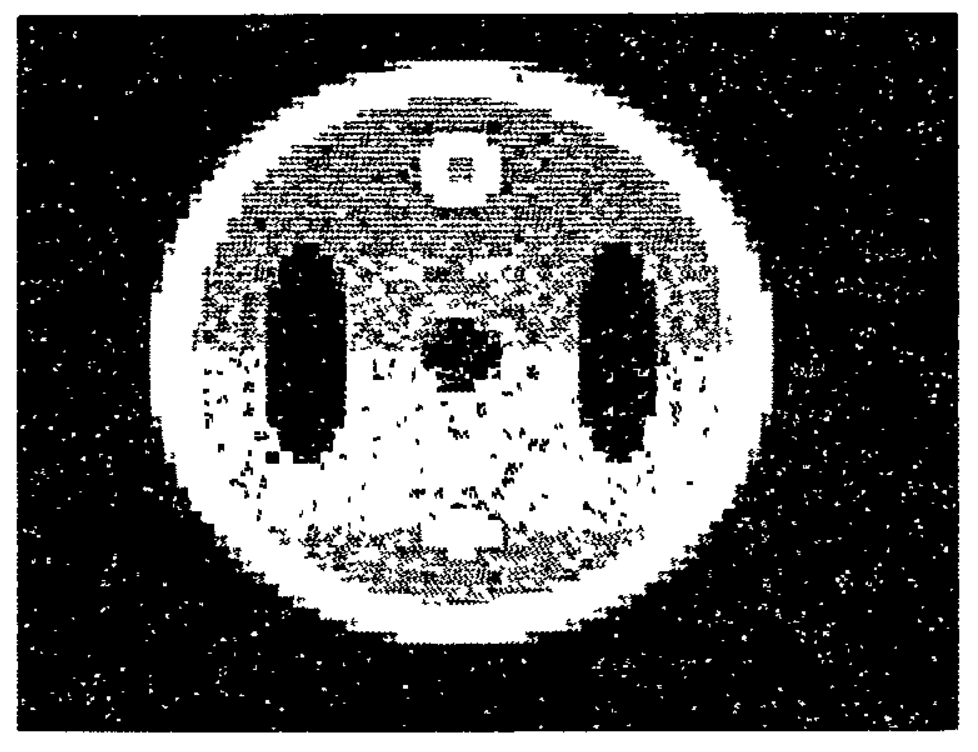

Flgure 130

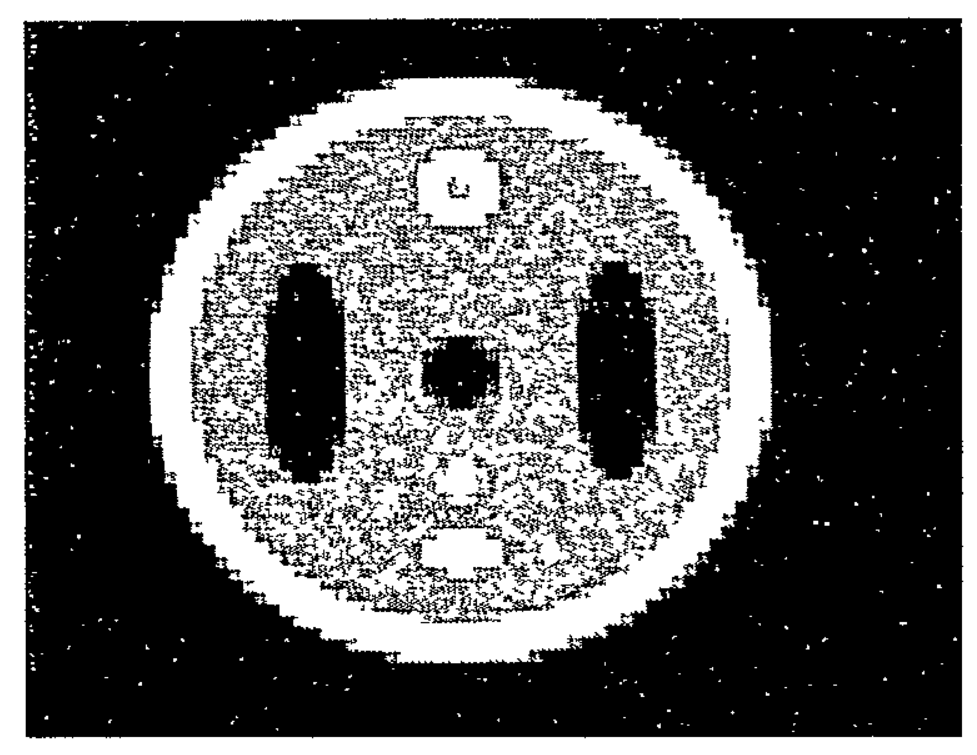

Flguxe $13 d$ 


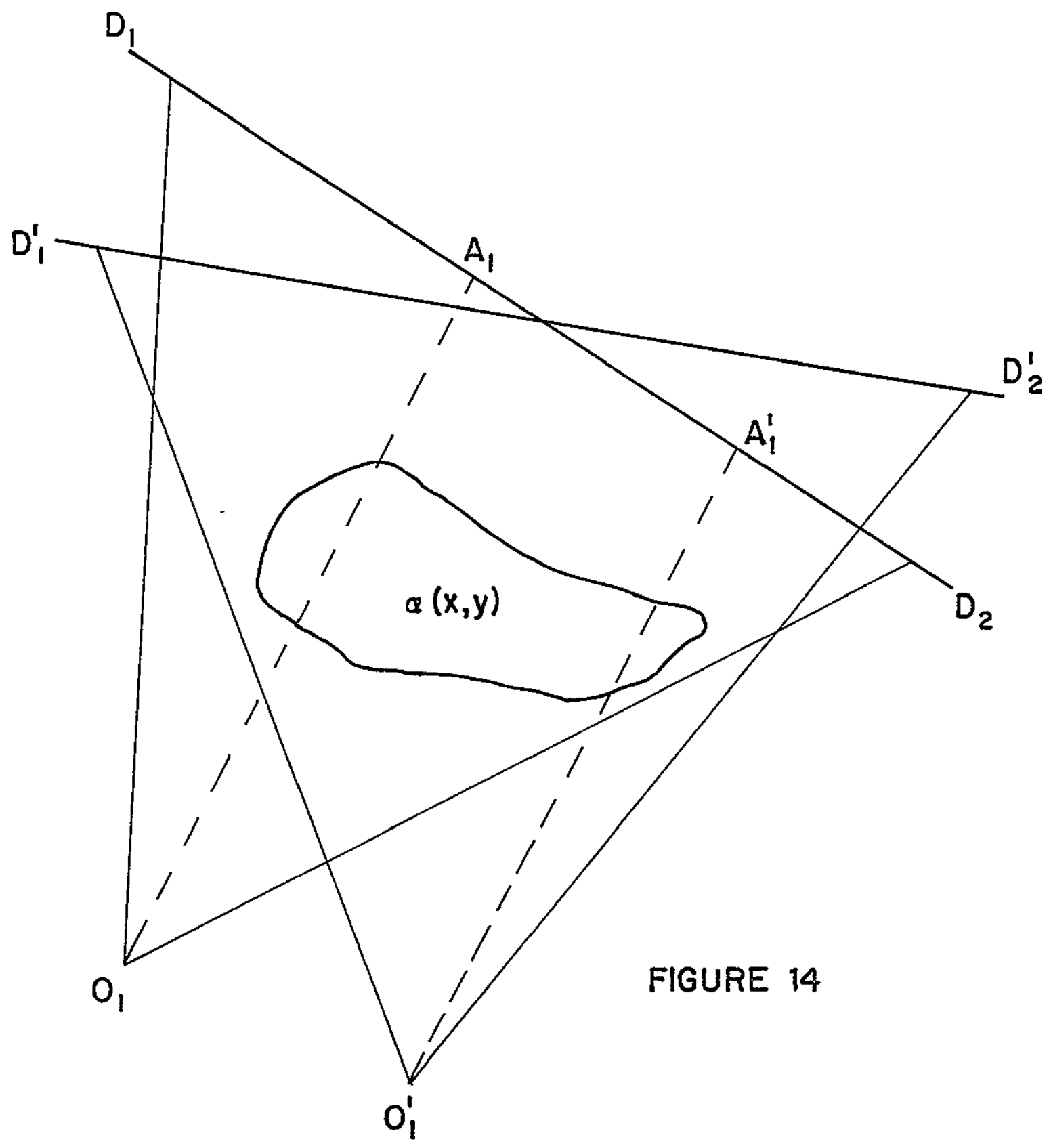




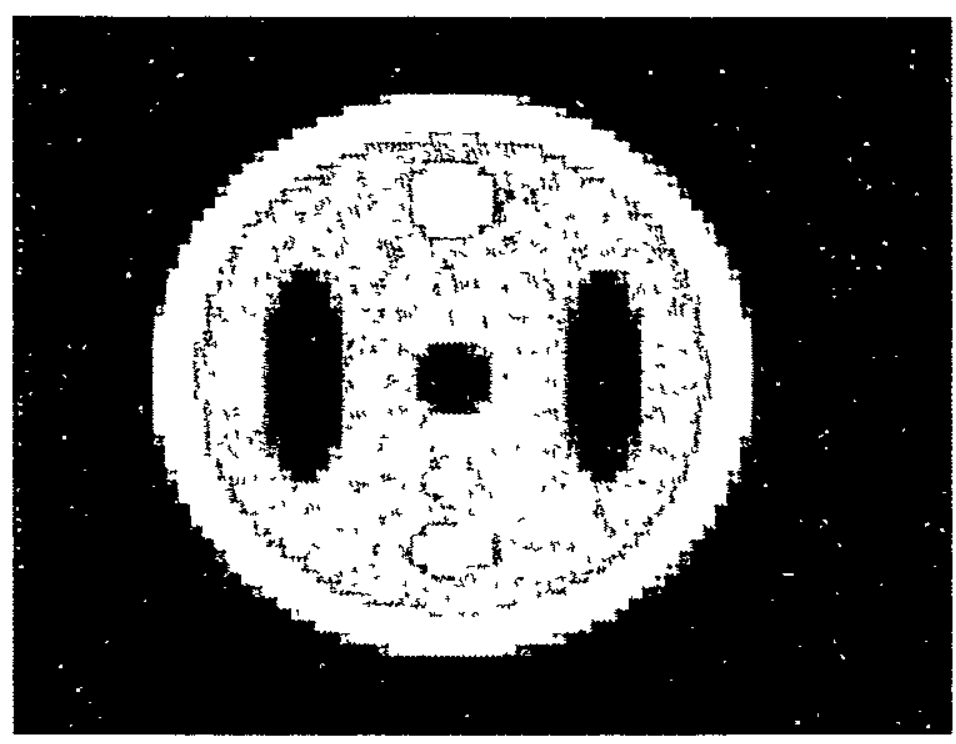

F1gare 150

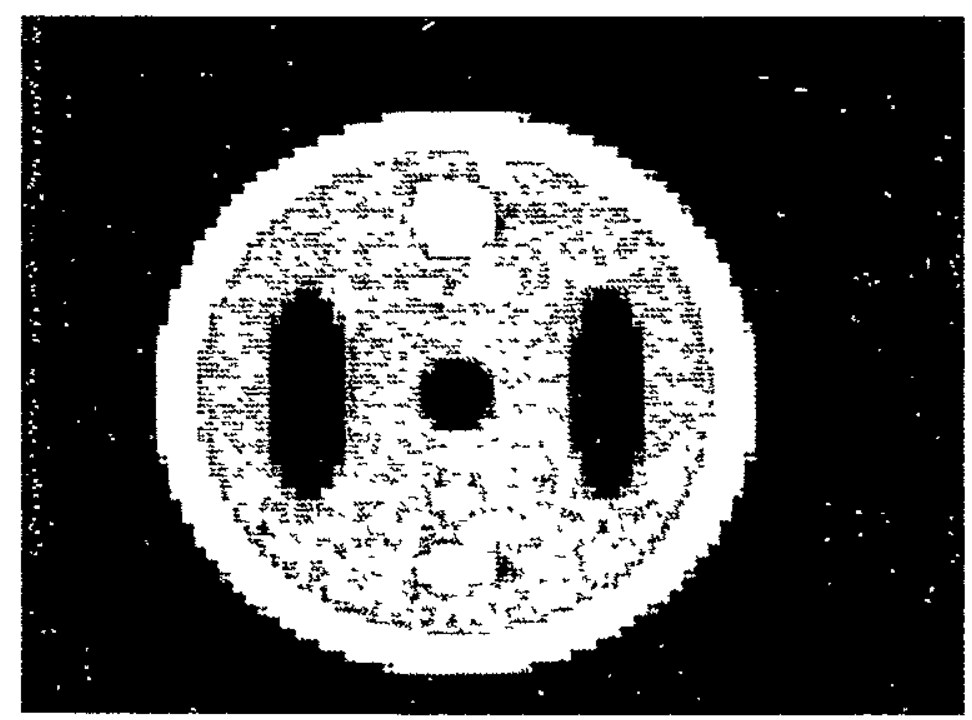

F1gure 150 


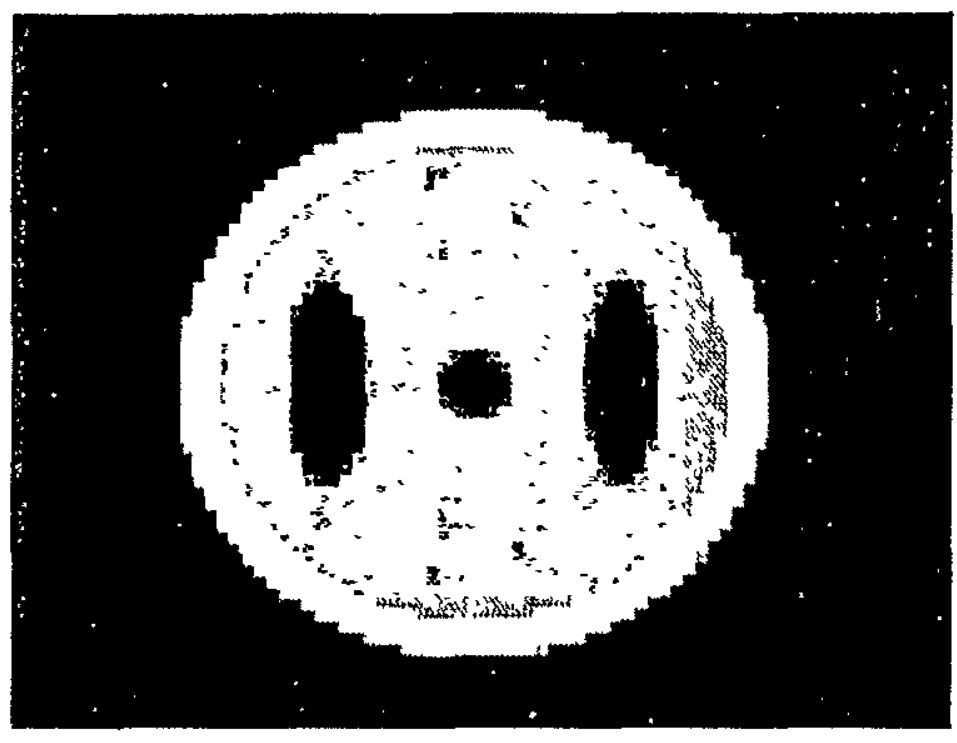

Figure $15 c$

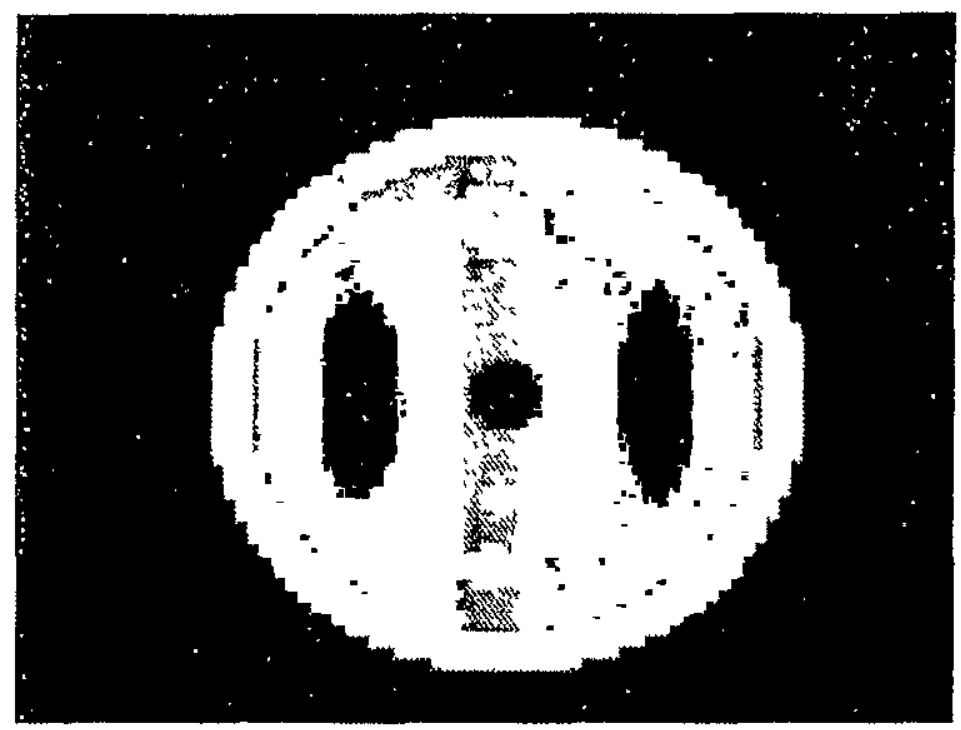

Figure 15d

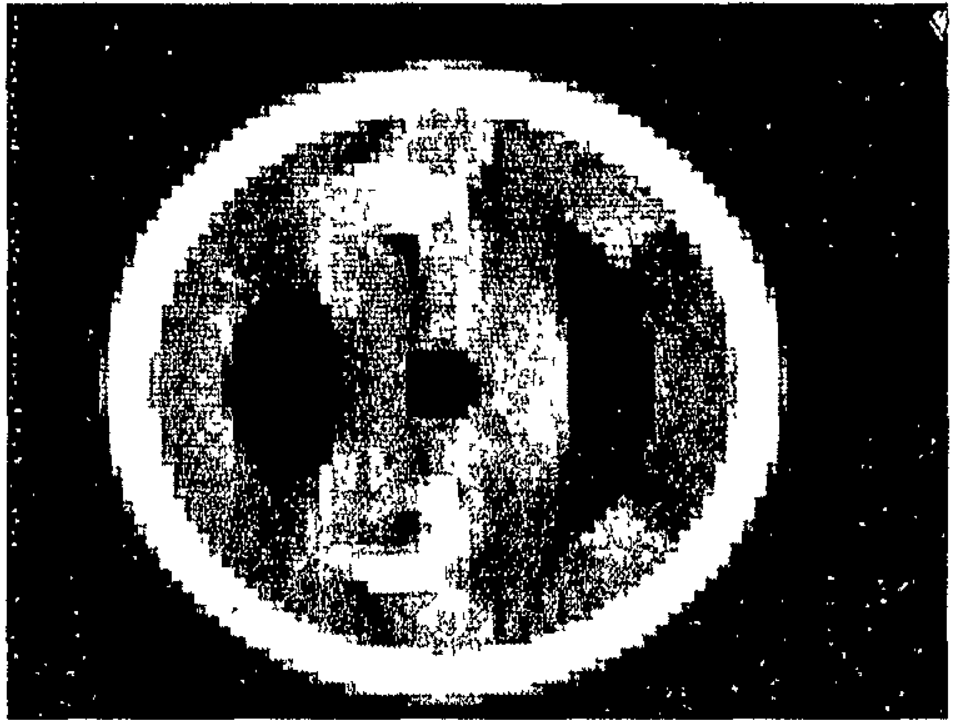

Flgure 150 


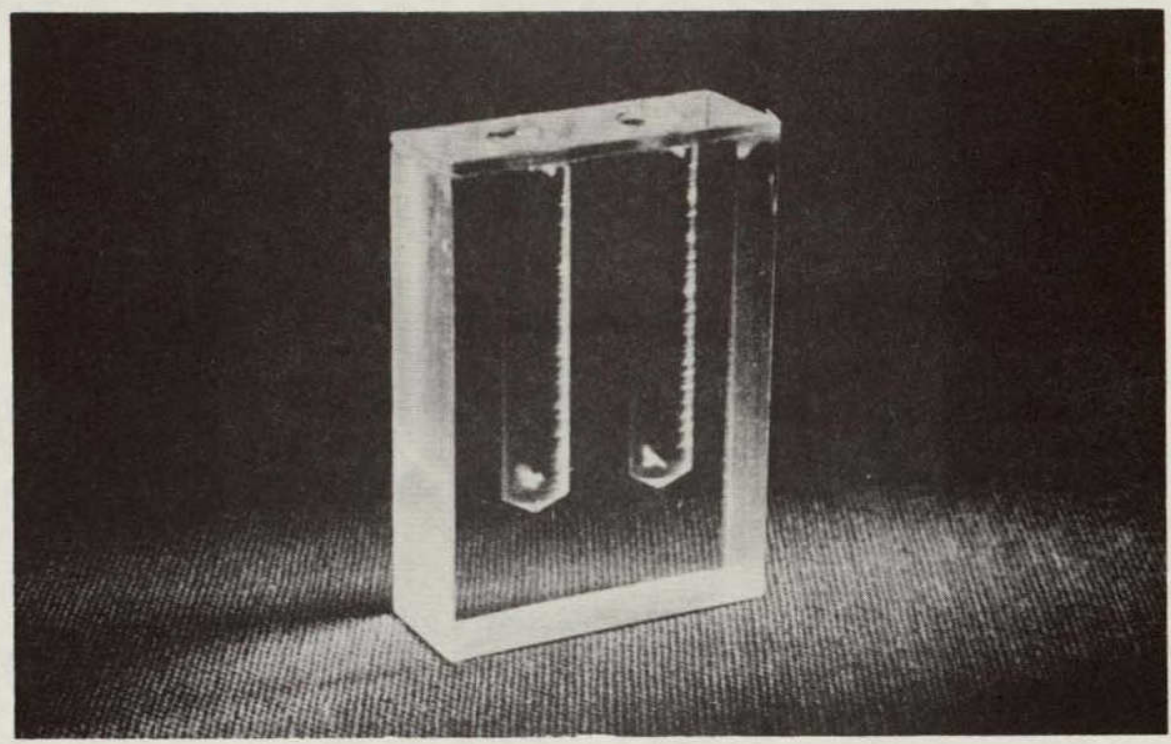

Figure $16 a$

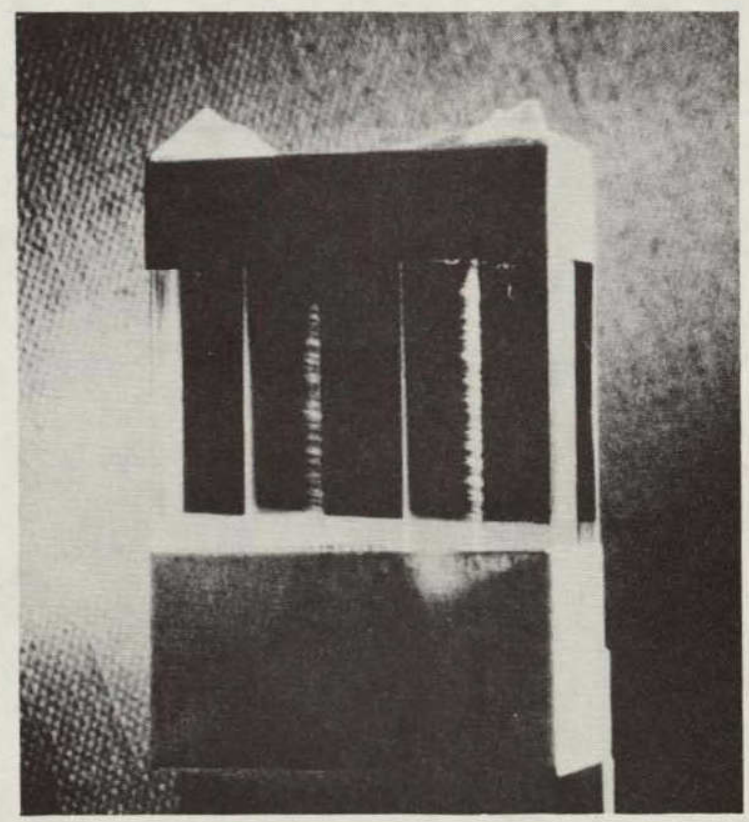

Figure 16b 


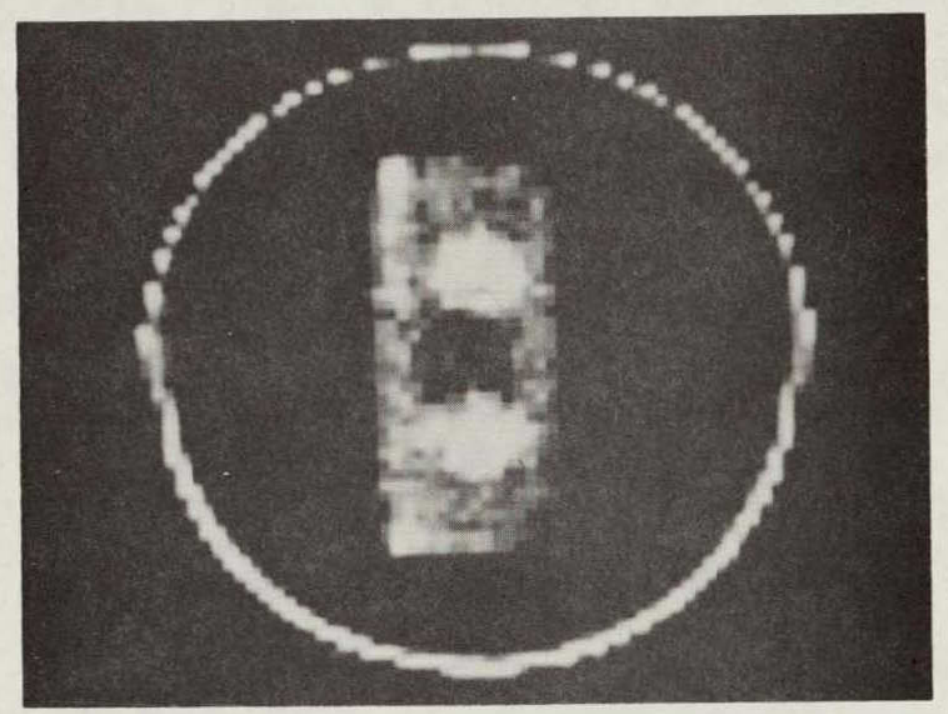

Figure $17 a$

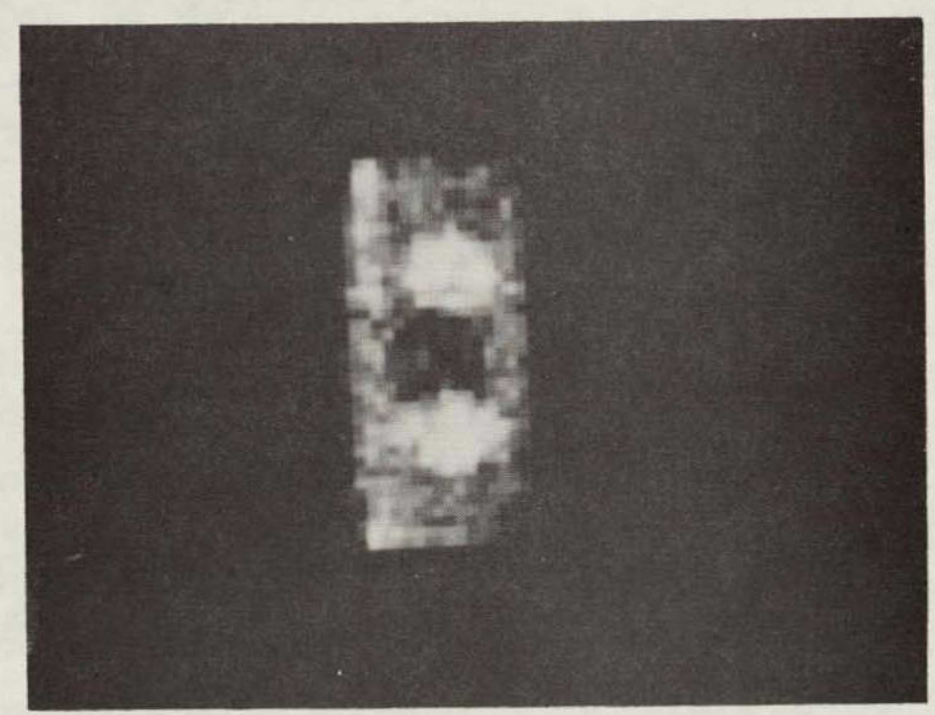

Figure 17b 


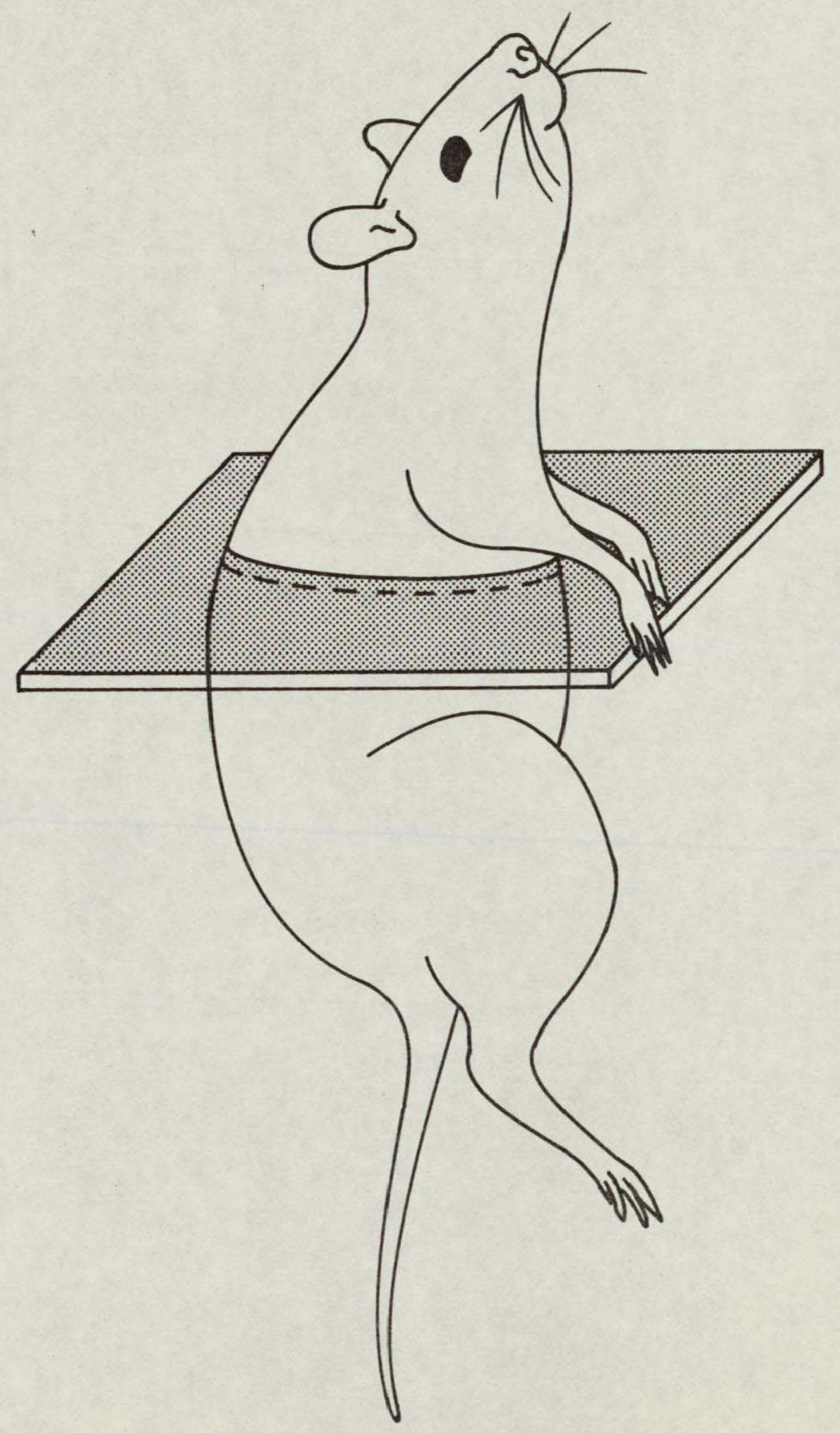

FIGURE 18a 


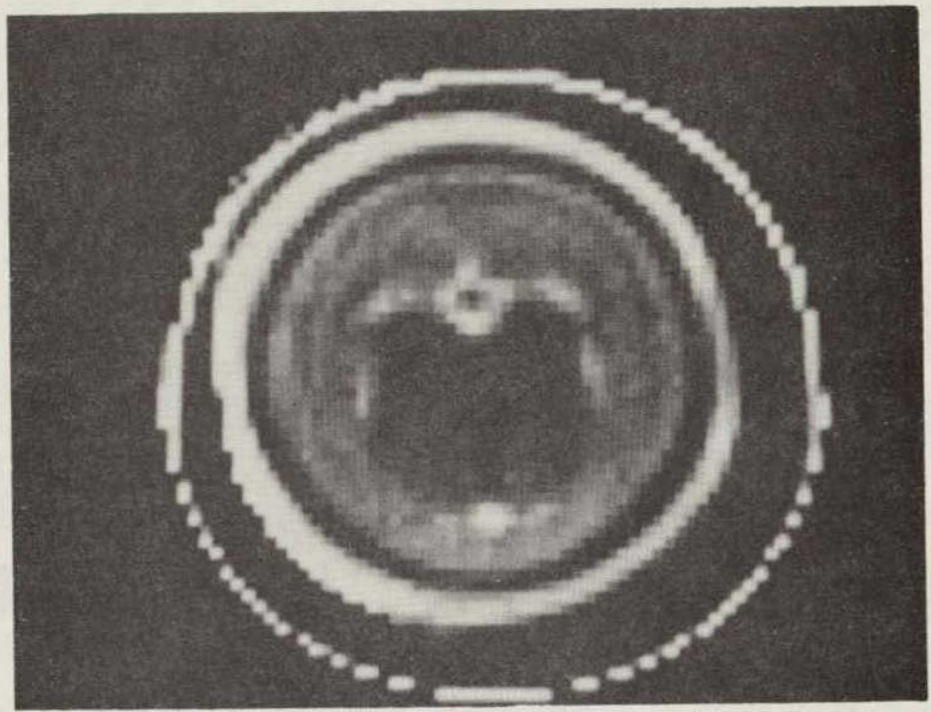

Figure $18 \mathrm{~b}$

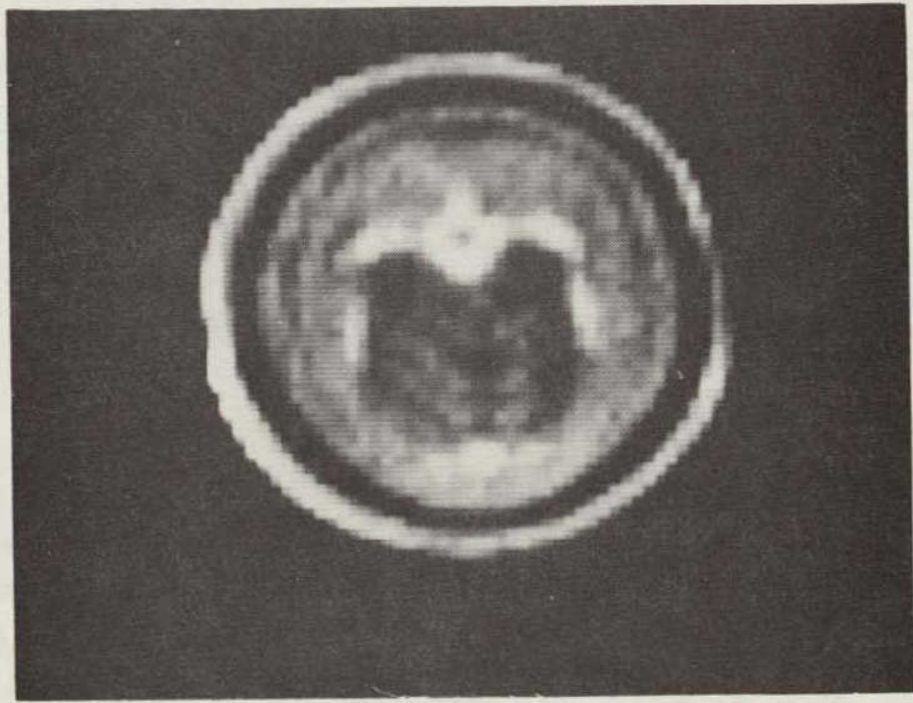

Figure $18 \mathrm{c}$ 
I. Spinal Column

2. Rib

3. Lungs

4. Heart

5. Sternum

6. Container

7. Air

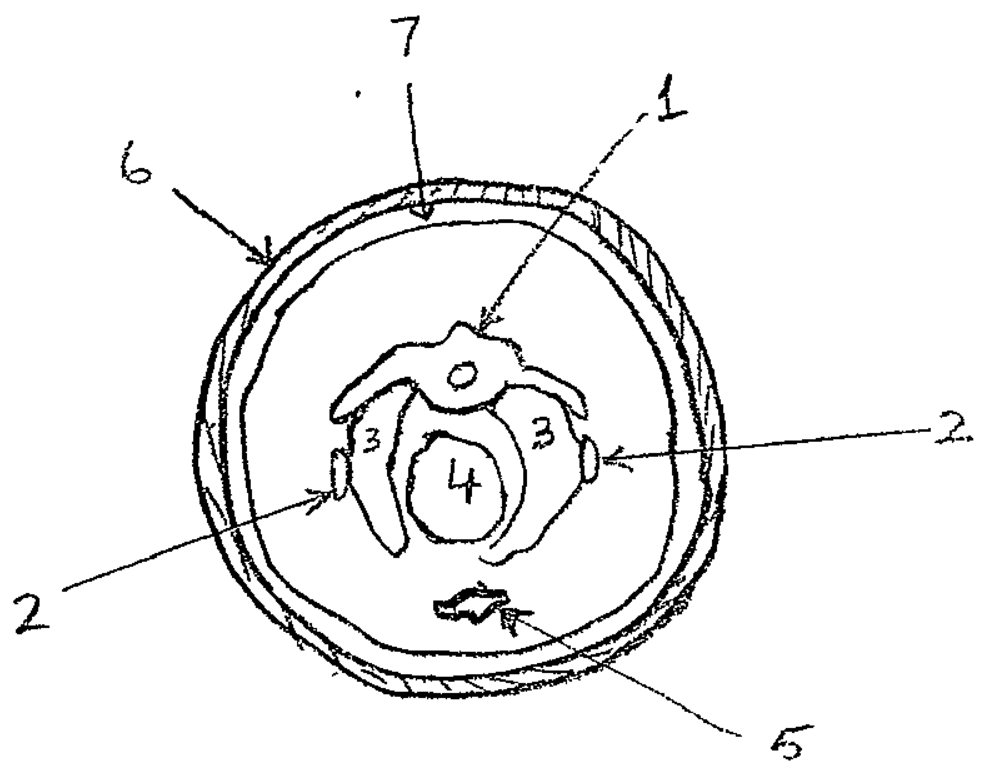

F/G. 18d 


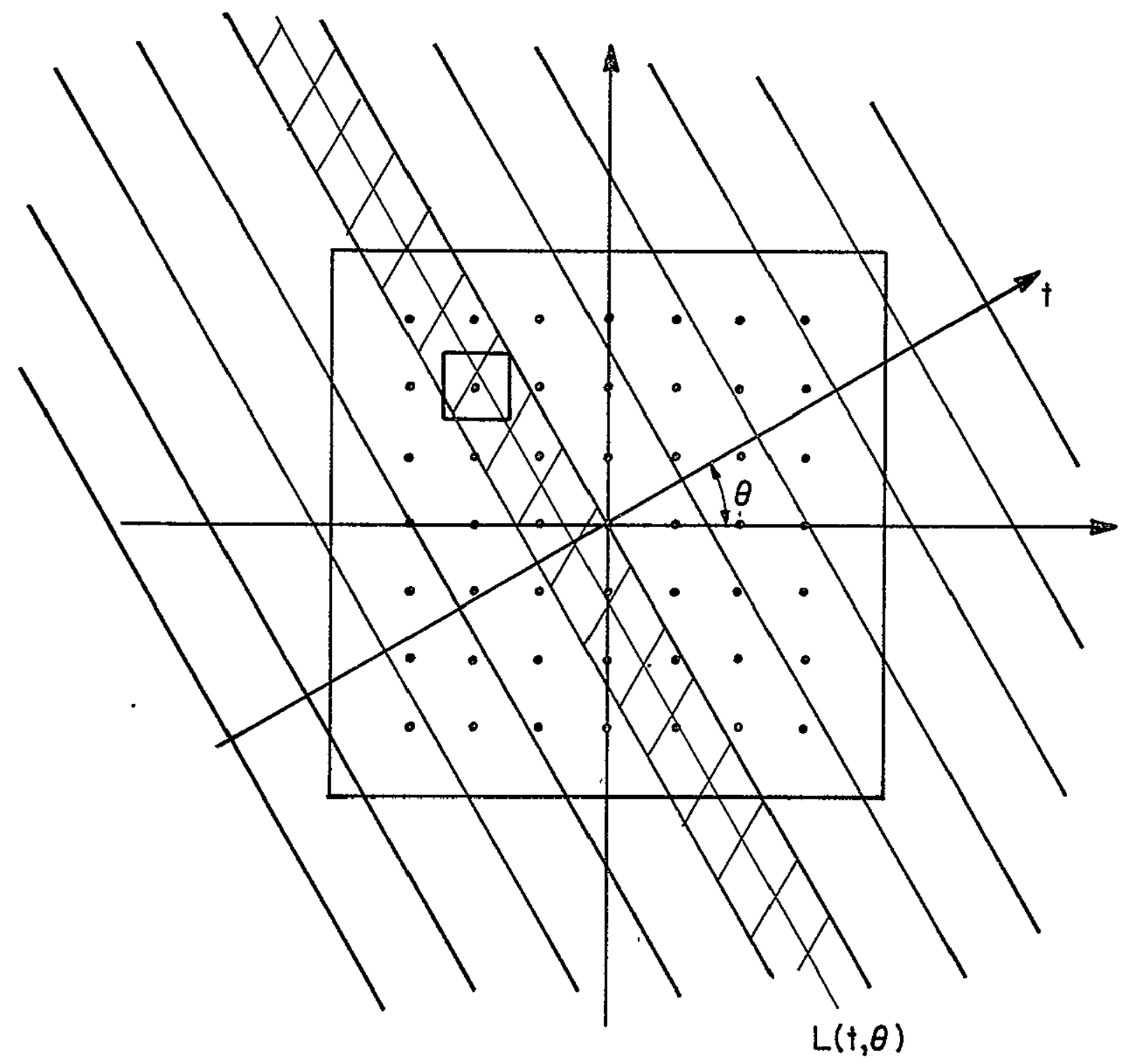

FIGURE A1.1 


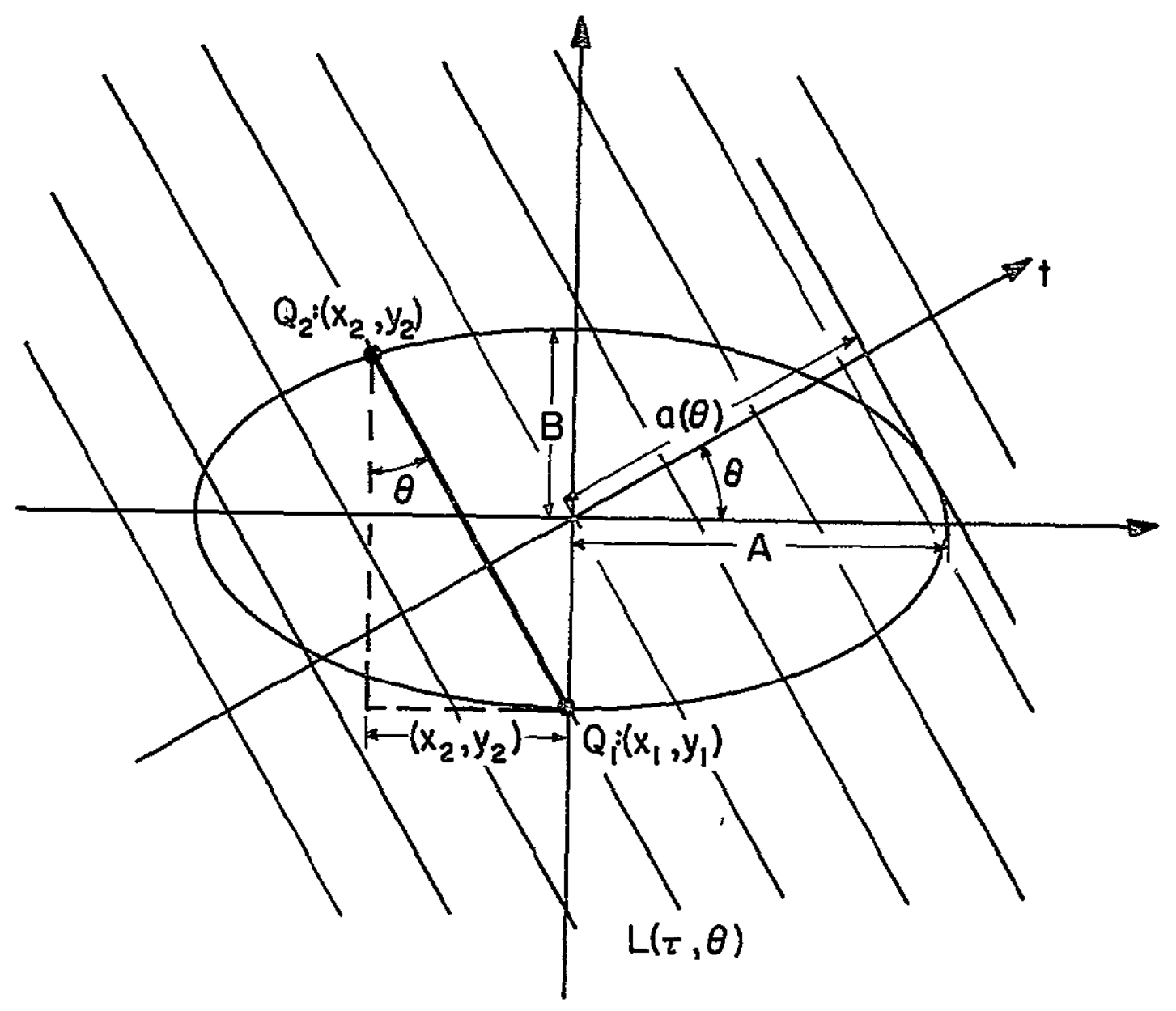

FIGURE A1.2 


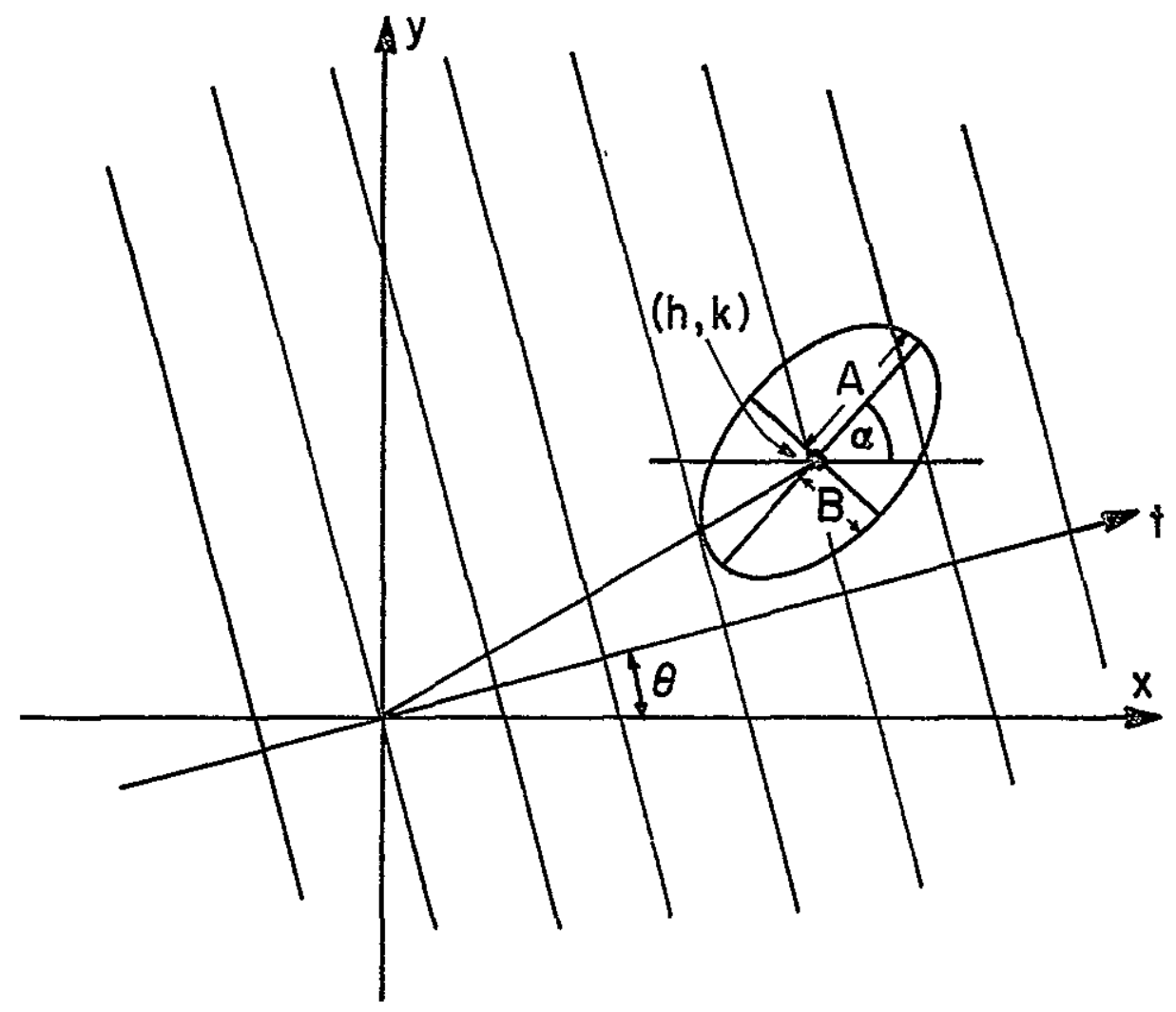

FIGURE A 1.3 


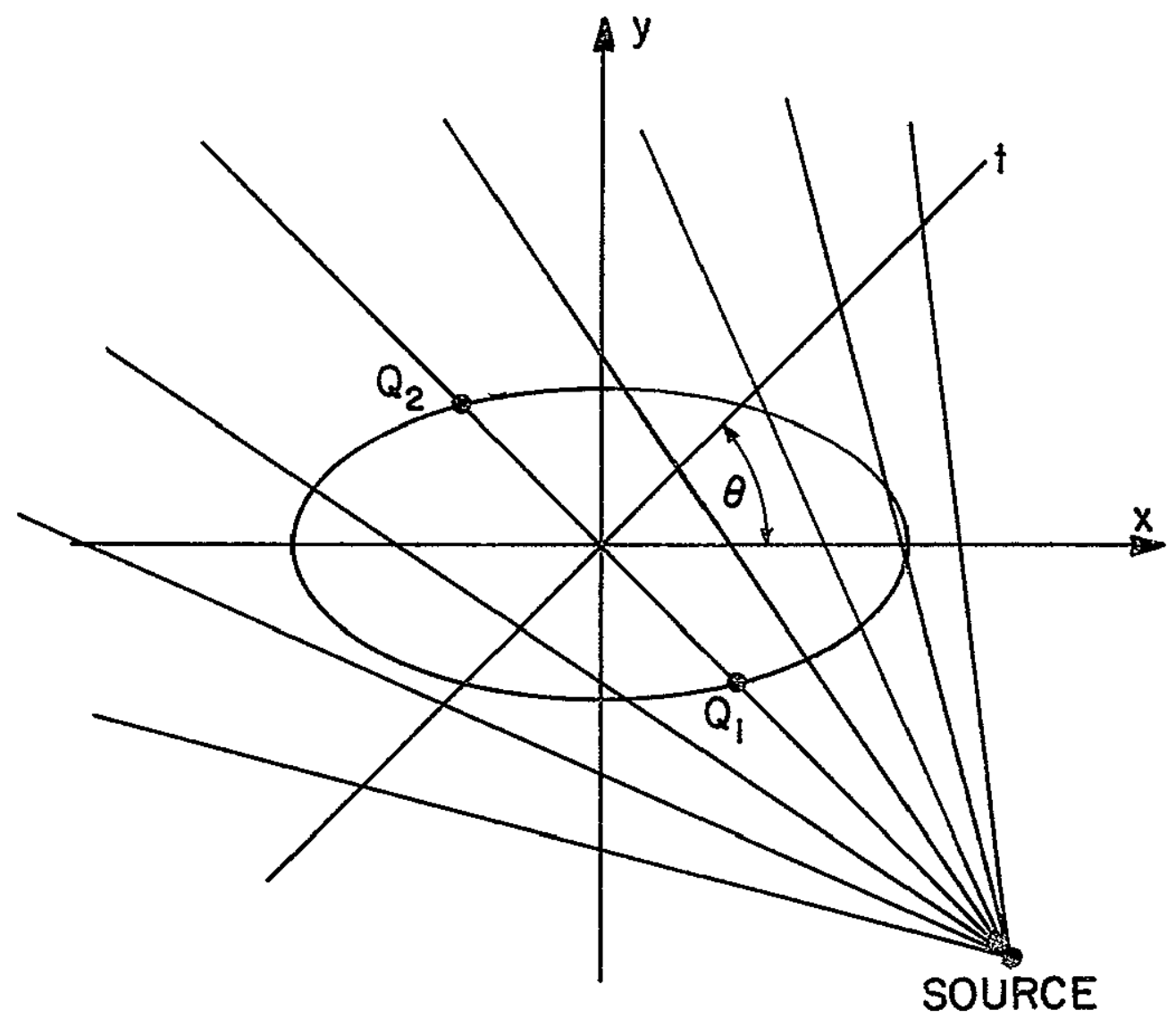

FIGURE A1.4 


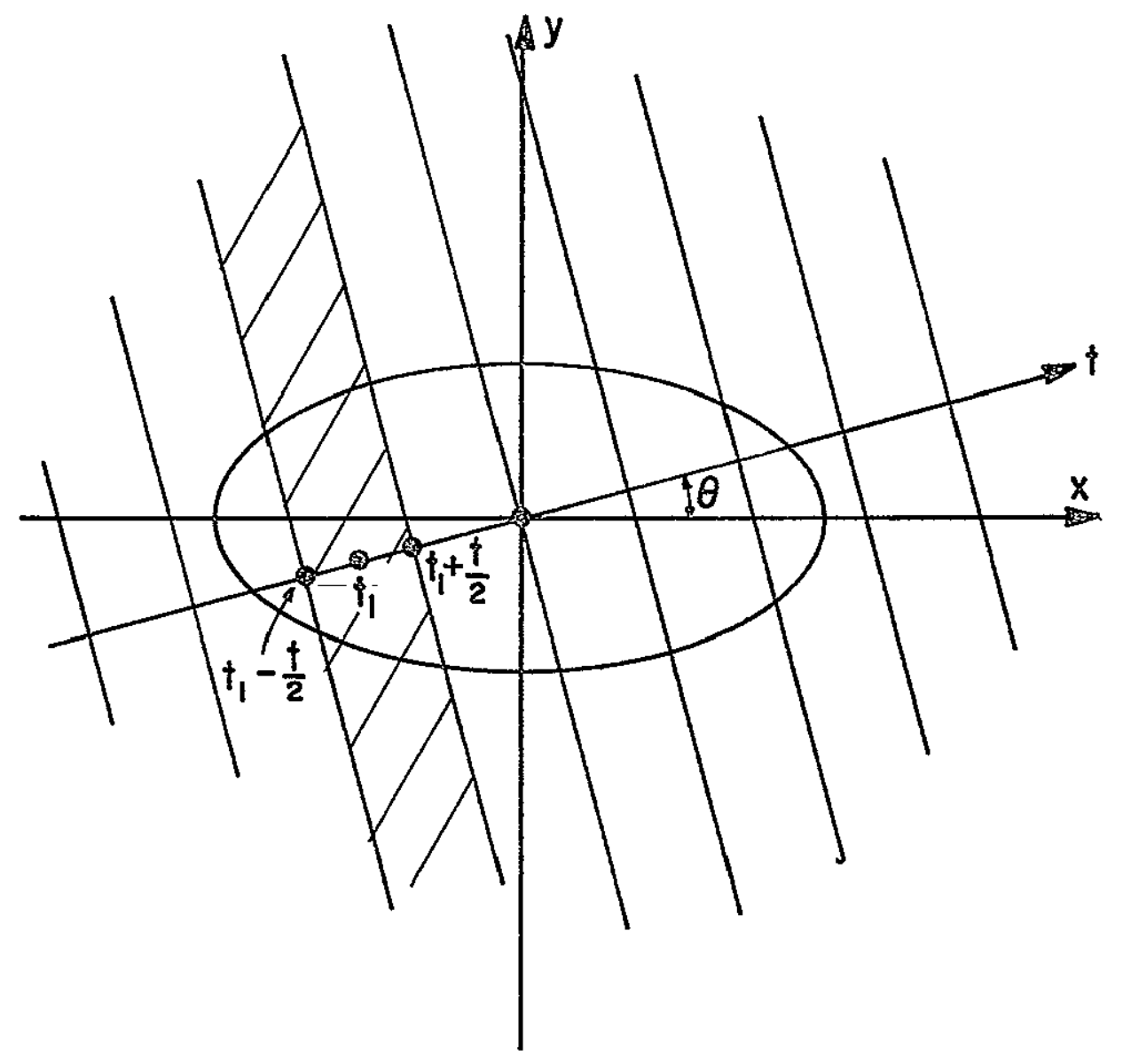

FIGURE A1.5 